\title{
Indonesian anthropometry update for special populations incorporating Drillis and Contini revisited
}

\author{
Markus Hartono \\ Laboratory of Engineering Management, Department of Industrial Engineering, University of Surabaya, Jalan Raya Kalirungkut, Tenggilis, Surabaya, 60293, \\ Indonesia
}

\section{A R T I C L E I N F O}

\section{Article history:}

Received 18 October 2016

Received in revised form

18 November 2017

Accepted 9 January 2018

\section{Keywords:}

Anthropometry

Special population

Drillis and Contini

Indonesian

\begin{abstract}
A B S T R A C T
Nowadays, research on anthropometry becomes more essential, and yet, it is critical due to its implication and contribution to product and system design. Since it deals with human capability and limitation on physical activities, its role becomes more important, especially, when it comes to the needs for special populations. This study provides a comparative study between elderly and children anthropometry using Drillis and Contini approach incorporating Chinese and non-Chinese ethnic groups. More than 1000 subjects involved in this study. After the data refinement process, there were 498 valid data for children (i.e., 98 Chinese male, 136 non-Chinese male, 134 Chinese female, and 130 non-Chinese female), and 556 valid data for elderly (i.e., 186 Chinese male, 148 non-Chinese male, 115 Chinese female, and 107 non-Chinese female).

In general, the finding shows that elderly (both male and female, both Chinese and non-Chinese) tends to have similar size and pattern with adult. Whilst, male and female children of 6-9 years sub-group (both Chinese and non-Chinese sub-group) tend to have higher weight ratio, compared to elderly and the children of 10-12 years sub-group. It was easily recognized that the children tend to have higher rate for limb segments compared to other body dimensions. At all sample groups, the eye height and shoulder height were found to be highly correlated with stature. Moreover, related to body weight, all samples show that thigh thickness and abdominal depth were deemed to be significant measures to be associated with.

The expected contribution of this study is that to update the Indonesian special population anthropometry and to identify which measures are significantly associated with stature and weight, respectively with regard to different special population and given limited anthropometric data. Practically, given the data of stature and body weight, product designer can predict the anthropometric characteristics for special population.
\end{abstract}

(C) 2018 Elsevier B.V. All rights reserved.

\section{Introduction}

Ergonomics deals with more humanized product, services and work systems. Due to rapid changing environment and system, human needs become more dynamic. Nevertheless, the users insist upon more efficient, comfortable, safe, healthy and effective working systems. Lack of it will lead to work-related injuries, low productivity, and product failures in market (Helander, 1997).

In dealing with physical human needs, anthropometry has been extensively proposed. It is not only discussing about how to measure and collect body measures, but also how to fit proper measures

E-mail address: markus@staff.ubaya.ac.id. into product characteristics and designs. The proper design of facilities and equipment utilizing the anthropometric data will increase productivity and reduce work-related musculoskeletal disorders (Klamklay et al., 2008; Chuan et al., 2010; Hartono and Gunawan, 2015).

Related to the basic Ergonomics, according to Kroemer and Grandjean (1997), anthropometry deals with and fits to human characteristics. Some pertinent factors such as genders, nutrition, and ethnic may influence the various result of human body dimensions. In work system design, it is called as user-centered design which involves the product, the user and the task (Hartono and Gunawan, 2015). It is in line with the ergonomics basic concept of how to fit the task to the human. By considering the anthropometric aspects, inherently, it will produce more 
efficient and productive work, as well as reduce any musculoskeletal disorders.

The challenge of anthropometry research is that how to maintain good consistency of measurement. It may deal with consistent measurement tools and facilities, prior knowledge of experimenter and researcher, and other environmental factors. It does not mean that the findings of anthropometric data should be of the same all the times. However, it is rather to achieve good fit data with minimum amount of errors. The data inconsistency may occur due to the positive or negative trend of anthropometric data with a function of time or other factors such as improved exercise, nutrition or newly life style, ethnic group, and special population. Special population may refer to children, elderly, pregnant women, and the ones who have different ability.

More specifically, Chuan et al. (2010) have conducted anthropometry study considering Indonesian and Singaporean populations, and compared the current data with the older one. In general, Singaporeans have been found to be larger than Indonesians. Both current Singaporean and Indonesian samples were also significantly larger than past Singaporeans and Indonesians, respectively. It might be caused by the change of geographical origin, nutrition, social status, and ethnic group. Assumed that there is no significant bias on the anthropometric measurement, thus, a regular update on anthropometric data is required. There is a potential need to deal with human anthropometric variability in designing any product or work systems.

When it reaches different users of different region such as countries, the first challenge is that how to deal with users from different countries, then it continues to controllable factors (e.g., nutrition, exercise, and social status). More specifically, anthropometric differences are not only found among different countries, but also among different ethnic groups within countries. Ethnic differences become the important precursor of various anthropometric measures. According to Widyanti et al. (2015), the anthropometric measurement taking into account different nationalities and ethnic groups has been conducted, such as Norwegian (Bolstad et al., 2001), Algerian (Mokdad, 2002), Portuguese (Barroso et al., 2005), Polish (Jarosz, 1999), Mexican American (Pennathura and Dowling, 2003), Indian (Victor et al., 2002), Filipinos (Del PradoLu, 2007), Swedish (Hanson et al., 2009), Bangladeshi (Khadem and Islam, 2014), and Iranian (Ghaderi et al., 2014; Sadeghi et al., 2015). Moreover, anthropometry study has been conducted in clinical practice to quantify changes in the craniofacial framework considering more than 25 ethnic groups (Leslie et al., 2005). The findings have shown that different ethnic groups have their distinctive anthropometric measures.

Regarding the concern on special population, a study on the effect of added weight on foot anthropometry in pregnant women by McCrory et al. (2016) has found that adding weight has brought significant changes in arch drop and arch rigidity index. Though, another factor such as the change of hormone concentrations should be taken into account. Another interesting study has been conducted by Harih and Dolšak (2013), which is about hand anthropometry for hand-operated tool. Its practical contribution is that the incorporation of the shape of hand during the optimal power-grasp posture. Significantly, it has improved the handle productivity. Related to children anthropometry, Jones et al. (2014) have conducted cross-country anthropometric study on height-forage z-score (HAZ) and weight-for-height z-score (WHZ). The quality of child diets and population trends were deemed to be sensitive concerns. Moreover, Olafsdottir et al. (2014) have done a longitudinal study describing the relationship between dietary habits, TV time and anthropometric measures for children. It showed that TV viewing activities have influenced the consumption of sugary drinks, and been followed by the increase in BMI and central obesity. Regarding the application of anthropometry for special population, a study on adult Special Olympics participants has been conducted by Temple et al. (2014). It was to explore the influence of gender and age on the body mass index (BMI). Interestingly, in general, it has been found that adult Special Olympics subjects have high level of BMI. In a wider coverage of application of anthropometric data, it might be applied into public facility. For instance, a recent study by Bombais et al. (2017) showed that the anthropometric data of adult were deemed critical to the bus layout and environment. Another interesting study on anthropometry for special population has been conducted by Widyanti et al. (2017). This study involved more than 600 Sundanese children anthropometric data (aged 6-9 years) in determining the clothing size. Moreover, study by Hartono (2016) which involved more than 400 subjects has found that limb segment and girth/width segment have a significant correlation with stature and body weight, respectively, through structural equation modeling (SEM).

With regard to Indonesia, a country with hundreds of ethnic groups, a research on the ethnic-based anthropometry for special population is relatively unexplored. A study by Chuan et al. (2010) has compared the Indonesian and Singaporean populations, and taken deeper analysis on the different ethnic groups (i.e., Chinese descendant and local citizen) within each country. A recent similar study by Widyanti et al. (2015) provides comparative anthropometric data among three major ethnic groups of Indonesian (i.e., Javanese, Sundanese and Minangkabau). This study, however, only discussed on Indonesian adult anthropometry. Practically, users are comprised of children, adult and elderly. Due to special need, influenced by growth period, elderly and children are called and adopted as the special population. Thus, it is of critical in the sense that how to satisfy their fitness and comfort when experiencing any product or working system. For example, due to aging, the diseases, disabilities and body function-decline will increase. More specifically, there are some challenges such as physical, social, and psychological functions that regard to performance-oriented assessment of mobility (Tinetti, 1986). Whilst, children have difficulty in fitting their capability to commonly used products and systems. For instance, Cadman et al. (1987) conducted a study of 3294 children of 4-16 years old with concern on chronic illness, physical disability and psychosocial problems. Both children and elderly have their specific and unique characteristics.

By considering different special populations (i.e., children and elderly) incorporating two different ethnic groups (i.e., Chinese and non-Chinese), this study is proposed to compare the differences in anthropometric measures. This current study can be considered a new study, but not necessarily the first one, made for special population in Indonesia. The previous study of anthropometry for special population in Indonesia has been conducted by Hartono (2016). The main difference is on the method used, number of samples and the depth of analyses. Following a study by Chuan et al. (2010), Chinese is regarded as the common ethnic group in the Southeast Asian region. Non-Chinese group will refer to the local citizen of a particular country. In Indonesia, the proportion of Chinese is around 5 percents, out of the country's total population.

By utilizing Drillis and Contini approach (Peacock et al., 2012), it is also to identify which measures are significantly associated with stature and weight, respectively with regard to different special population and ethnic groups. It is expected that the results of this study can be utilized as a general guide in predicting reliable and valid anthropometric measures considering different population and ethnic groups, with respect to the limited sample size. 


\section{Literature review}

\subsection{Special populations and ethnic groups in anthropometry}

Special populations require special treatment. Since it is of uncommon population group, anthropometry should accommodate its unique characteristics and differences. They are facing potential problems, due to their limited abilities and needs. It may imply the challenge of how to design tools and tasks (Kroemer, 2006). The special populations differ from their peers in strength, posture and size, and sensing and predicting abilities. According to Kroemer (2006), more specifically, elderly as one of the special populations needs special attention in terms of body size and posture, physical abilities, and psychological traits change. Moreover, children may draw more attention as the younger the children are, the more different they are from the adult.

Compared to adults as a common group, special population which consists of children and elderly groups may have different anthropometric dimensions, in terms of the size and ratio between measures. In order to fit the facilities and equipment to specific population group, thus, selection process and utilization of anthropometric database is crucial and critical (Wickens et al., 2004). It implies that the detailed characteristics of anthropometric data should be of critical part in any anthropometry studies. A study by Widyanti et al. (2015) has confirmed the significant difference among different ethnic groups. Some demographic factors such as ethnic origin, social status, sufficient nutrition and geographical origin were shown significantly in influencing the differences on anthropometric measures. It was then followed by the different measures between male and female subjects. Male was found to be bigger and taller than female anthropometric data (Widyanti et al., 2015; Chuan et al., 2010). It implies that diversified ethnic groups within a particular population may bring significant differences in anthropometric data. More specifically, ethnic differences should be considered in any system design process.

\subsection{Drillis and Contini revisited}

According to Peacock et al. (2012) and Drillis and Contini (1966), several body segments are inter-correlated. Through a single measure of stature (body height), the other body dimensions have been hypothesized that they can be predicted sufficiently and accurately (Drillis and Contini, 1966; Pheasant and Haslegrave, 2006). Their findings have been published and applied (Peacock et al., 2012). In order to improve the reliability of Drillis and Contini's prediction model, a study done by Peacock et al. (2012) showed that width and girth measures were inter-correlated and more associated with body weight, than body height. Thus, apart from stature, the addition of body weight serves greater accuracy.

Body height and weight are commonly used as the reference points. It means that they can be used as predictors to other measures. Width and girth measures had more significant correlation with body weight, whereas, stature has been set to be a predictor for limb length.

In short, according to Peacock et al. (2012), limb segments are significantly related to the whole-body density. Recent research on Indonesian adult anthropometry shows that several body measures are significantly inter-correlated (see Hartono and Gunawan, 2015). Stature and body weight are deemed to be the most basic body segments for predicting other measures. Related to the previous study in 2010 (Chuan et al., 2010), the current Indonesian anthropometric measures were relatively higher and bigger than the old ones due to better income, education, nutrition and medical services. In short, the body dimensions which are significantly associated either with stature or weight can be identified (Hartono and
Gunawan, 2015). Subsequently, it helps product designers to fit the body measures to particular physical product or any human-work system interactions more effectively and efficiently.

\section{Research methodology}

This study and data collection has been carried out over a period of 24 months (in a range of 2015-2016). It applied convenience sampling to select and arrange the subjects. Anthropometry research is one of the very sensitive research forms dealing with human. Hence, it is quite challenging, subjects may refuse or disregard our invitation to join the experiment. Due to this consideration on the ease of subject accessibility and approach, the convenience sampling has been chosen. The intended subjects were selected based on their availability and willingness to respond. To avoid any potential biases, the experimenter has filtered the subjects by asking their willingness to join the survey and confirming the criteria required. Gravetter and Forzano (2012) pointed out that there is a strategy to reduce bias by ensuring that the subjects are reasonably representative and not strongly biased. It means that the subjects should be the representative of the population of interest. This strategy has been used in this current study as well. All samples have been carefully selected according to the intended main criteria (i.e., Indonesian children aged 6-12 years, and Indonesian elderly aged 55 years and above).

According to Chuan et al. (2010), the data collection on body dimensions is a very sensitive, thus, some respondents may refuse. The potential subjects were approached and asked whether they were willing to participate in the proposed anthropometric study. Some potential questions and doubts asked by the subjects have been directly responded. It has been continued until everything has been settled, and agreed by the subjects. If the subjects refused, the experimenters left and the measurement was cancelled.

\subsection{Subjects}

As for children subjects, the experimenter asked permission from their parents or teachers, whereas, for elderly, the experimenter asked directly to the subjects and or the owner of elderly house where they were staying. In total, more than 1000 subjects involved in the study. There were 498 children aged 6-12 years (i.e., 98 Chinese male, 136 non-Chinese male, 134 Chinese female, and 130 non-Chinese female), and 556 elderly aged 55 years and above (i.e., 186 Chinese male, 148 non-Chinese male, 115 Chinese female, and 107 non-Chinese female). Due to a relatively wide range of ages, more specifically, the children group was divided into two age groups (i.e., 6-9 years and 10-12 years) at each of the ethnic groups (i.e., Chinese and non-Chinese). Moreover, in order to increase data validity by excluding any uncontrollable measurements, the x-bar control chart was utilized. According to BPS (2015), the numbers of Indonesian children aged 6-12 years and elderly aged 55 years and above were 32,433,444 and 26,474,282 people, respectively. According to Altares et al. (2003), by using Sloven's formula $\left[n=N /\left(1+N^{*} e^{2}\right)\right.$, where: $n=$ minimum number of samples, $\mathrm{N}=$ total population, and $\mathrm{e}=$ error tolerance] with an error tolerance of $5 \%$, the expected minimum number of children and elderly samples were 399 and 400, respectively. Hence, the samples of both children and elderly taken in this study were deemed sufficient. In addition to the elderly subjects, there was no one had serious health problems during the experiment.

\subsection{Procedure}

Subjects were barefooted, wearing t-shirts and pants, and measured using 36 body dimensions as modified and used by 


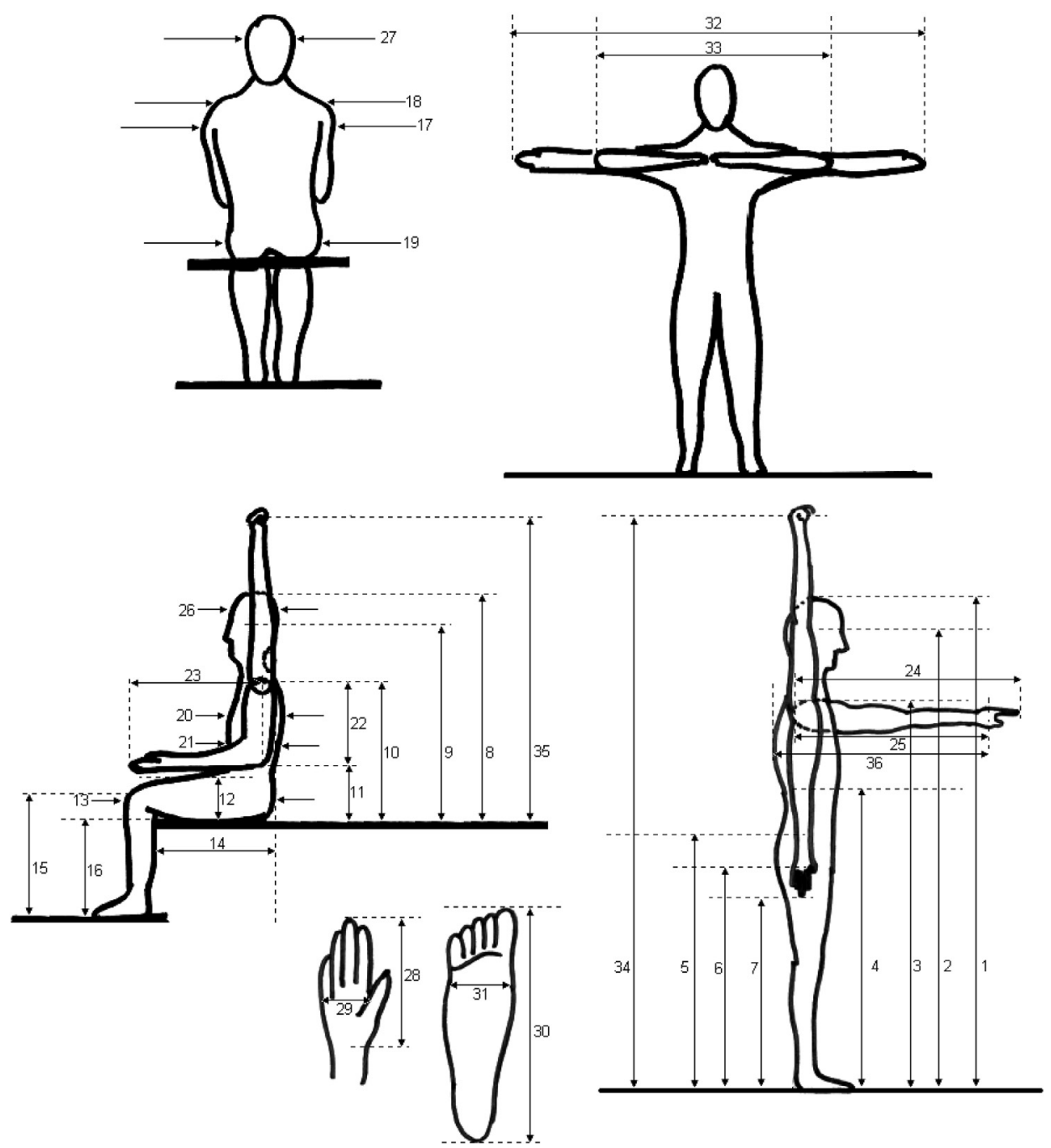

Fig. 1. Thirty six body landmarks (modified from Chuan et al., 2010).

[Note: Numbers shown in Fig. 1 show the names of body dimension as available in Table 1].

Chuan et al. (2010). Ideally, each section of data collection, there were at least two experimenters involved. However, in a special case, there was only one experimenter getting involved due to either the time or appointment constraint with the prospective subject. As a consequence, the experiment took longer. In addition, it is when the subject deals with the experimenter regardless the gender issue, thus, the experiment will be carried on. Hence, the male experiment may have an opportunity to do measurement to female subject, and vice versa. In the case of two experimenters, one took the measurement and the other one recorded the data. All those activities are shown in Figs. 2 and 3. In order to minimize any potential human errors during data collection process, all experimenters have been selected through the evaluation process conducted by the representative of ergonomics laboratory in the
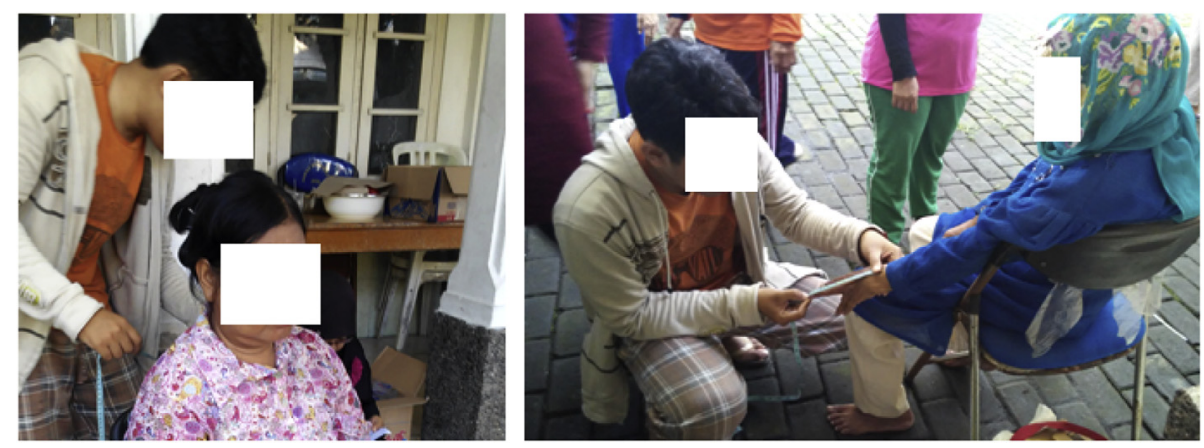

Fig. 2. Measurement of elderly by 1 experimenter. 


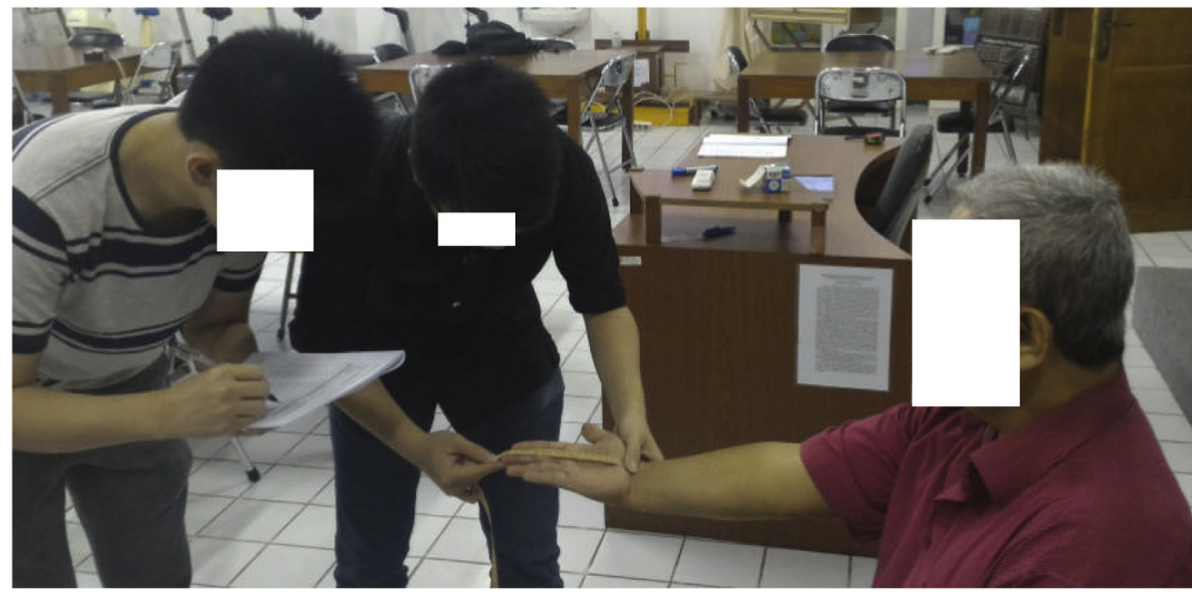

Fig. 3. Measurement of elderly by 2 experimenters.

university and equipped with the knowledge on how to use the equipment, body landmarks, and recognize the bony tips that influence the validity of measured figures. The experimenters have conducted several practices and pilot tests to get engaged with the experiment and data collection process.

In addition, once the anthropometric data have been collected, the identification of outlier data was conducted through the use of $\mathrm{x}$-bar control chart. It is to identify the causes of variation to investigate, and to decide whether we need to exclude the uncontrollable data (Montgomery et al., 2010). Thus, the final data set is hoped to be stable and valid.

Table 1

Anthropometric data for 6-9 years old children with subgroups of Chinese and non-Chinese (all dimensions in cm).

\begin{tabular}{|c|c|c|c|c|c|c|c|c|c|c|c|c|c|c|c|c|}
\hline \multirow[t]{3}{*}{ Dimension } & \multicolumn{8}{|l|}{ Male } & \multicolumn{8}{|c|}{ Female } \\
\hline & \multicolumn{4}{|c|}{ Chinese } & \multicolumn{4}{|c|}{ non-Chinese } & \multicolumn{4}{|c|}{ Chinese } & \multicolumn{4}{|c|}{ non-Chinese } \\
\hline & 5 th & 50th & 95th & SD & 5 th & 50th & 95th & SD & 5 th & 50th & 95th & SD & 5 th & 50th & 95th & SD \\
\hline 1. Stature & 100.7 & 117.8 & 134.9 & 10.4 & 104.6 & 121.7 & 138.9 & 10.4 & 108.1 & 121.9 & 135.6 & 8.4 & 89.5 & 122.8 & 156.2 & 20.3 \\
\hline 2. Eye height & 80.7 & 101.2 & 121.8 & 12.5 & 93.0 & 111.3 & 129.5 & 11.1 & 96.7 & 110.0 & 123.3 & 8.1 & 89.3 & 116.4 & 143.5 & 16.5 \\
\hline 3. Shoulder height & 72.4 & 90.2 & 107.9 & 10.8 & 79.3 & 99.3 & 119.3 & 12.2 & 83.0 & 97.6 & 112.1 & 8.8 & 75.3 & 101.1 & 126.9 & 15.7 \\
\hline 4. Elbow height & 54.6 & 68.1 & 81.6 & 8.2 & 62.2 & 76.6 & 90.9 & 8.7 & 65.6 & 74.7 & 83.8 & 5.5 & 60.3 & 84.6 & 109.0 & 14.8 \\
\hline 5. Hip height & 43.5 & 59.2 & 74.9 & 9.5 & 51.0 & 67.8 & 84.6 & 10.2 & 56.7 & 67.0 & 77.3 & 6.3 & 49.2 & 74.1 & 99.0 & 15.1 \\
\hline 6. Knuckel height & 35.4 & 46.1 & 56.9 & 6.5 & 40.5 & 51.0 & 61.6 & 6.4 & 43.1 & 50.8 & 58.6 & 4.7 & 42.9 & 57.6 & 72.3 & 8.9 \\
\hline 7. Fingertip height & 29.5 & 39.3 & 49.1 & 5.9 & 34.8 & 44.4 & 54.0 & 5.8 & 38.9 & 43.8 & 48.7 & 3.0 & 36.9 & 51.7 & 66.5 & 9.0 \\
\hline 8. Sitting height & 52.4 & 61.0 & 69.7 & 5.2 & 54.4 & 64.9 & 75.4 & 6.4 & 55.2 & 63.0 & 70.8 & 4.7 & 52.8 & 67.0 & 81.2 & 8.6 \\
\hline 9. Sitting eye height & 40.3 & 52.1 & 64.0 & 7.2 & 41.7 & 53.4 & 65.0 & 7.1 & 47.3 & 54.0 & 60.7 & 4.1 & 43.6 & 59.0 & 74.4 & 9.4 \\
\hline 10. Sitting shoulder height & 26.4 & 37.2 & 48.0 & 6.6 & 29.9 & 41.9 & 53.9 & 7.3 & 34.5 & 41.1 & 47.7 & 4.0 & 29.4 & 43.5 & 57.5 & 8.6 \\
\hline 11. Sitting elbow height & 9.7 & 14.9 & 20.2 & 3.2 & 7.9 & 15.5 & 23.1 & 4.6 & 10.6 & 16.8 & 22.9 & 3.7 & 7.9 & 17.8 & 27.8 & 6.1 \\
\hline 12. Thigh thickness & 5.7 & 10.3 & 14.9 & 2.8 & 5.2 & 9.9 & 14.6 & 2.8 & 5.7 & 8.8 & 11.9 & 1.9 & 5.2 & 9.9 & 14.6 & 2.8 \\
\hline 13. Buttock-knee length & 25.3 & 35.2 & 45.1 & 6.0 & 30.0 & 40.2 & 50.5 & 6.2 & 34.8 & 40.0 & 45.1 & 3.2 & 30.4 & 43.7 & 57.0 & 8.1 \\
\hline 14. Buttock-popliteal length & 20.5 & 29.5 & 38.5 & 5.5 & 25.5 & 34.5 & 43.6 & 5.5 & 29.0 & 34.2 & 39.4 & 3.2 & 27.0 & 38.3 & 49.6 & 6.9 \\
\hline 15. Knee height & 28.4 & 37.7 & 47.0 & 5.7 & 27.8 & 40.2 & 52.5 & 7.5 & 31.9 & 42.2 & 52.6 & 6.3 & 29.1 & 44.4 & 59.7 & 9.3 \\
\hline 16. Popliteal height & 24.4 & 31.4 & 38.4 & 4.2 & 25.4 & 33.1 & 40.8 & 4.7 & 25.7 & 32.7 & 39.7 & 4.3 & 25.3 & 36.7 & 48.0 & 6.9 \\
\hline 17. Shoulder breadth (bideltoid) & 24.4 & 29.9 & 35.4 & 3.4 & 24.5 & 32.1 & 39.8 & 4.6 & 25.4 & 31.2 & 37.0 & 3.5 & 20.5 & 35.9 & 51.4 & 9.4 \\
\hline 18. Shoulder breadth (biacromial) & 14.9 & 22.7 & 30.5 & 4.8 & 14.3 & 25.7 & 37.2 & 7.0 & 16.1 & 24.3 & 32.5 & 5.0 & 13.6 & 28.4 & 43.1 & 9.0 \\
\hline 19. Hip breadth & 17.7 & 24.1 & 30.6 & 3.9 & 16.7 & 26.5 & 36.3 & 5.9 & 19.7 & 26.0 & 32.4 & 3.9 & 14.5 & 29.3 & 44.1 & 9.0 \\
\hline 20. Chest (bust) depth & 10.3 & 14.6 & 18.9 & 2.6 & 8.2 & 14.6 & 21.1 & 3.9 & 10.7 & 15.1 & 19.4 & 2.6 & 6.4 & 15.8 & 25.3 & 5.7 \\
\hline 21. Abdominal depth & 10.8 & 16.0 & 21.3 & 3.2 & 9.5 & 17.4 & 25.2 & 4.8 & 12.5 & 16.7 & 20.8 & 2.5 & 7.0 & 17.5 & 27.9 & 6.3 \\
\hline 22. Shoulder-elbow length & 16.2 & 22.5 & 28.8 & 3.8 & 14.4 & 25.2 & 36.1 & 6.6 & 18.2 & 24.6 & 30.9 & 3.9 & 10.7 & 25.0 & 39.3 & 8.7 \\
\hline 23. Elbow-fingertip length & 23.2 & 30.0 & 36.9 & 4.2 & 25.2 & 33.6 & 42.0 & 5.1 & 27.3 & 31.8 & 36.4 & 2.8 & 17.2 & 35.9 & 54.7 & 11.4 \\
\hline 24. Upper limb length & 35.3 & 48.3 & 61.3 & 7.9 & 40.5 & 52.1 & 63.6 & 7.0 & 42.3 & 50.7 & 59.0 & 5.1 & 34.6 & 52.8 & 71.0 & 11.1 \\
\hline 25. Shoulder-grip length & 26.3 & 37.7 & 49.1 & 6.9 & 32.1 & 43.3 & 54.6 & 6.8 & 35.0 & 42.3 & 49.6 & 4.5 & 31.8 & 48.7 & 65.7 & 10.3 \\
\hline 26. Head length & 11.8 & 16.3 & 20.7 & 2.7 & 12.7 & 17.8 & 23.0 & 3.1 & 14.0 & 16.7 & 19.4 & 1.7 & 14.0 & 18.6 & 23.2 & 2.8 \\
\hline 27. Head breadth & 9.6 & 15.8 & 22.0 & 3.8 & 9.8 & 16.8 & 23.9 & 4.3 & 11.9 & 17.2 & 22.5 & 3.2 & 8.1 & 19.2 & 30.3 & 6.8 \\
\hline 28. Hand length & 9.6 & 12.4 & 15.2 & 1.7 & 10.6 & 13.9 & 17.2 & 2.0 & 11.4 & 14.2 & 17.1 & 1.7 & 10.3 & 15.8 & 21.2 & 3.3 \\
\hline 29. Hand breadth & 4.8 & 6.1 & 7.4 & 0.8 & 4.8 & 6.6 & 8.4 & 1.1 & 5.3 & 6.6 & 8.0 & 0.8 & 3.3 & 6.2 & 9.1 & 1.8 \\
\hline 30. Foot length & 13.0 & 17.1 & 21.2 & 2.5 & 14.7 & 19.3 & 23.8 & 2.8 & 15.6 & 19.3 & 22.9 & 2.2 & 14.2 & 19.3 & 24.4 & 3.1 \\
\hline 31. Foot breadth & 4.3 & 6.8 & 9.2 & 1.5 & 5.3 & 7.7 & 10.0 & 1.4 & 5.7 & 7.7 & 9.7 & 1.2 & 4.6 & 8.5 & 12.4 & 2.4 \\
\hline 32. Span & 85.2 & 108.2 & 131.3 & 14.0 & 99.7 & 122.2 & 144.6 & 13.7 & 99.9 & 118.0 & 136.1 & 11.0 & 95.5 & 130.3 & 165.2 & 21.2 \\
\hline 33. Elbow span & 42.4 & 61.8 & 81.1 & 11.8 & 47.7 & 63.4 & 79.1 & 9.5 & 52.4 & 61.7 & 71.1 & 5.7 & 47.0 & 67.7 & 88.4 & 12.6 \\
\hline 34. Vertical grip reach (standing) & 107.1 & 132.1 & 157.1 & 15.2 & 118.3 & 146.1 & 173.8 & 16.9 & 123.9 & 143.4 & 162.8 & 11.8 & 119.3 & 160.0 & 200.8 & 24.8 \\
\hline 35. Vertical grip reach (sitting) & 63.4 & 83.9 & 104.4 & 12.5 & 69.1 & 86.6 & 104.1 & 10.6 & 70.1 & 81.8 & 93.4 & 7.1 & 60.0 & 86.9 & 113.9 & 16.4 \\
\hline 36. Forward grip reach & 31.2 & 45.3 & 59.4 & 8.6 & 36.7 & 51.6 & 66.4 & 9.0 & 38.3 & 49.9 & 61.5 & 7.1 & 38.1 & 59.8 & 81.5 & 13.2 \\
\hline
\end{tabular}


Table 2

Anthropometric data for 10-12 years old children with subgroups of Chinese and non-Chinese (all dimensions in $\mathrm{cm}$ ).

\begin{tabular}{|c|c|c|c|c|c|c|c|c|c|c|c|c|c|c|c|c|}
\hline \multirow[t]{3}{*}{ Dimension } & \multicolumn{8}{|l|}{ Male } & \multicolumn{8}{|c|}{ Female } \\
\hline & \multicolumn{4}{|c|}{ Chinese } & \multicolumn{4}{|c|}{ non-Chinese } & \multicolumn{4}{|c|}{ Chinese } & \multicolumn{4}{|c|}{ non-Chinese } \\
\hline & 5 th & 50th & 95th & SD & 5 th & 50th & 95th & SD & 5 th & 50th & 95th & SD & 5 th & 50th & 95th & SD \\
\hline 1. Stature & 124.1 & 139.1 & 154.2 & 9.2 & 123.9 & 140.3 & 156.7 & 10.0 & 124.2 & 141.0 & 157.7 & 10.2 & 111.4 & 137.0 & 162.6 & 15.5 \\
\hline 2. Eye height & 113.2 & 126.9 & 140.6 & 8.3 & 109.6 & 126.7 & 143.8 & 10.4 & 112.8 & 130.7 & 148.6 & 10.9 & 90.1 & 121.1 & 152.1 & 18.8 \\
\hline 3. Shoulder height & 98.5 & 112.3 & 126.2 & 8.4 & 92.0 & 112.3 & 132.6 & 12.3 & 98.3 & 113.6 & 129.0 & 9.3 & 76.6 & 104.0 & 131.4 & 16.7 \\
\hline 4. Elbow height & 75.6 & 87.0 & 98.5 & 7.0 & 66.6 & 84.7 & 102.8 & 11.0 & 75.3 & 87.2 & 99.1 & 7.2 & 61.4 & 85.0 & 108.5 & 14.3 \\
\hline 5. Hip height & 65.0 & 76.7 & 88.4 & 7.1 & 60.4 & 75.8 & 91.2 & 9.3 & 66.4 & 79.9 & 93.3 & 8.2 & 49.9 & 75.6 & 101.2 & 15.6 \\
\hline 6. Knuckel height & 48.9 & 59.5 & 70.2 & 6.5 & 44.6 & 58.4 & 72.2 & 8.4 & 50.3 & 60.1 & 70.0 & 6.0 & 41.2 & 56.2 & 71.2 & 9.1 \\
\hline 7. Fingertip height & 42.1 & 50.7 & 59.4 & 5.3 & 38.1 & 48.5 & 58.9 & 6.3 & 42.6 & 51.3 & 60.0 & 5.3 & 37.2 & 50.3 & 63.5 & 8.0 \\
\hline 8. Sitting height & 62.2 & 70.6 & 78.9 & 5.1 & 57.2 & 68.0 & 78.8 & 6.6 & 61.4 & 70.9 & 80.5 & 5.8 & 52.6 & 67.5 & 82.5 & 9.1 \\
\hline 9. Sitting eye height & 51.5 & 60.1 & 68.8 & 5.3 & 46.1 & 58.6 & 71.2 & 7.6 & 50.0 & 60.6 & 71.1 & 6.4 & 46.3 & 62.3 & 78.2 & 9.7 \\
\hline 10. Sitting shoulder height & 37.9 & 46.9 & 55.9 & 5.5 & 34.0 & 45.5 & 57.1 & 7.0 & 36.2 & 45.7 & 55.2 & 5.8 & 26.9 & 42.6 & 58.4 & 9.6 \\
\hline 11. Sitting elbow height & 12.0 & 18.4 & 24.8 & 3.9 & 9.9 & 17.5 & 25.0 & 4.6 & 11.0 & 18.1 & 25.2 & 4.3 & 7.9 & 18.0 & 28.0 & 6.1 \\
\hline 12. Thigh thickness & 6.4 & 10.7 & 15.0 & 2.6 & 5.3 & 11.8 & 18.2 & 3.9 & 7.1 & 10.9 & 14.7 & 2.3 & 5.9 & 10.7 & 15.5 & 2.9 \\
\hline 13. Buttock-knee length & 38.8 & 46.0 & 53.1 & 4.3 & 33.7 & 44.4 & 55.1 & 6.5 & 37.9 & 46.6 & 55.4 & 5.3 & 28.4 & 42.9 & 57.3 & 8.8 \\
\hline 14. Buttock-popliteal length & 31.0 & 39.3 & 47.6 & 5.1 & 27.8 & 37.5 & 47.2 & 5.9 & 32.8 & 39.6 & 46.4 & 4.1 & 27.4 & 37.7 & 47.9 & 6.2 \\
\hline 15. Knee height & 37.5 & 44.8 & 52.1 & 4.4 & 34.0 & 42.2 & 50.5 & 5.0 & 36.5 & 44.4 & 52.3 & 4.8 & 30.4 & 45.1 & 59.9 & 9.0 \\
\hline 16. Popliteal height & 32.3 & 37.5 & 42.8 & 3.2 & 28.5 & 36.8 & 45.0 & 5.0 & 29.9 & 37.5 & 45.0 & 4.6 & 25.3 & 36.1 & 46.9 & 6.5 \\
\hline 17. Shoulder breadth (bideltoid) & 26.3 & 34.4 & 42.4 & 4.9 & 23.5 & 33.8 & 44.0 & 6.2 & 25.4 & 35.5 & 45.6 & 6.1 & 21.6 & 35.4 & 49.3 & 8.4 \\
\hline 18. Shoulder breadth (biacromial) & 20.4 & 28.5 & 36.6 & 4.9 & 16.6 & 27.8 & 38.9 & 6.8 & 18.0 & 29.2 & 40.5 & 6.8 & 13.2 & 26.6 & 40.1 & 8.2 \\
\hline 19. Hip breadth & 21.9 & 31.1 & 40.2 & 5.5 & 19.3 & 27.8 & 36.4 & 5.2 & 20.0 & 29.8 & 39.6 & 5.9 & 16.5 & 30.5 & 44.4 & 8.5 \\
\hline 20. Chest (bust) depth & 12.0 & 17.0 & 22.0 & 3.1 & 10.8 & 16.5 & 22.2 & 3.5 & 10.2 & 16.1 & 21.9 & 3.5 & 9.0 & 17.1 & 25.2 & 4.9 \\
\hline 21. Abdominal depth & 10.5 & 17.8 & 25.1 & 4.5 & 11.9 & 19.2 & 26.5 & 4.5 & 9.4 & 16.9 & 24.5 & 4.6 & 6.4 & 16.9 & 27.5 & 6.4 \\
\hline 22. Shoulder-elbow length & 22.4 & 29.0 & 35.6 & 4.0 & 18.4 & 28.6 & 38.8 & 6.2 & 22.4 & 29.0 & 35.6 & 4.0 & 13.1 & 26.3 & 39.5 & 8.0 \\
\hline 23. Elbow-fingertip length & 31.2 & 37.7 & 44.2 & 3.9 & 30.2 & 37.4 & 44.6 & 4.4 & 31.1 & 38.8 & 46.4 & 4.6 & 18.2 & 34.0 & 49.7 & 9.6 \\
\hline 24. Upper limb length & 53.4 & 60.8 & 68.2 & 4.5 & 47.8 & 59.9 & 72.1 & 7.4 & 50.9 & 61.3 & 71.7 & 6.4 & 35.0 & 51.7 & 68.3 & 10.1 \\
\hline 25. Shoulder-grip length & 39.7 & 48.6 & 57.5 & 5.4 & 38.2 & 50.7 & 63.1 & 7.6 & 41.9 & 51.6 & 61.4 & 5.9 & 29.6 & 47.1 & 64.6 & 10.6 \\
\hline 26. Head length & 14.1 & 18.3 & 22.5 & 2.6 & 13.0 & 18.3 & 23.7 & 3.3 & 13.7 & 17.7 & 21.7 & 2.4 & 12.9 & 18.2 & 23.5 & 3.2 \\
\hline 27. Head breadth & 13.4 & 17.4 & 21.5 & 2.5 & 9.8 & 17.2 & 24.6 & 4.5 & 11.3 & 18.5 & 25.7 & 4.4 & 9.0 & 18.8 & 28.6 & 5.9 \\
\hline 28. Hand length & 13.0 & 15.7 & 18.5 & 1.7 & 11.2 & 15.2 & 19.2 & 2.4 & 12.6 & 16.1 & 19.7 & 2.2 & 9.4 & 14.7 & 19.9 & 3.2 \\
\hline 29. Hand breadth & 5.8 & 7.2 & 8.6 & 0.9 & 5.1 & 7.0 & 8.9 & 1.2 & 5.1 & 7.2 & 9.3 & 1.3 & 3.8 & 7.1 & 10.3 & 2.0 \\
\hline 30. Foot length & 18.5 & 21.7 & 24.9 & 1.9 & 15.7 & 21.9 & 28.0 & 3.8 & 18.3 & 21.2 & 24.2 & 1.8 & 14.4 & 20.3 & 26.2 & 3.6 \\
\hline 31. Foot breadth & 6.7 & 8.9 & 11.1 & 1.3 & 6.3 & 8.4 & 10.6 & 1.3 & 6.3 & 8.6 & 10.8 & 1.4 & 4.8 & 8.5 & 12.1 & 2.2 \\
\hline 32. Span & 123.0 & 137.5 & 151.9 & 8.8 & 113.5 & 136.3 & 159.1 & 13.9 & 123.2 & 141.4 & 159.7 & 11.1 & 93.0 & 129.3 & 165.5 & 22.0 \\
\hline 33. Elbow span & 59.9 & 70.0 & 80.1 & 6.2 & 54.5 & 70.4 & 86.3 & 9.7 & 61.2 & 72.8 & 84.3 & 7.0 & 49.1 & 68.0 & 86.9 & 11.5 \\
\hline 34. Vertical grip reach (standing) & 148.4 & 164.0 & 179.5 & 9.4 & 138.0 & 166.7 & 195.4 & 17.4 & 143.8 & 167.5 & 191.2 & 14.4 & 118.9 & 156.0 & 193.1 & 22.6 \\
\hline 35. Vertical grip reach (sitting) & 82.4 & 94.1 & 105.7 & 7.1 & 76.0 & 93.4 & 110.7 & 10.6 & 80.1 & 96.1 & 112.1 & 9.7 & 59.8 & 85.7 & 111.6 & 15.7 \\
\hline 36. Forward grip reach & 45.5 & 56.4 & 67.2 & 6.6 & 43.4 & 57.3 & 71.2 & 8.5 & 47.5 & 59.9 & 72.3 & 7.5 & 37.2 & 59.0 & 80.9 & 13.3 \\
\hline
\end{tabular}

\subsection{Body landmarks and measurement equipment}

There were 36 body dimensions, body weight, and the classification of Chinese or non-Chinese, utilized. All those body dimensions were specified by Pheasant and Haslegrave (2006) and used by Chuan et al. (2010).

By adapting what has been utilized by Chuan et al. (2010) and Al-Ansari and Mokdad (2009), a convenient anthropometric tape, small stool, weighing scale have been used. It is due to simple, portable and inexpensive consideration, and yet, it is effective and efficient. To ensure more effective, efficient and less bias result, the experimenters and helpers were well trained. The subjects must have been asked to maintain proper posture, yet natural, during the period of measurement. It took about 20-30 min to complete one subject. Ideally, to promote privacy and code of conduct, since it is so sensitive, male experimenter has been responsible for male respondents, whereas female experimenter has taken care of female respondents, unless the subjects agree with any experimenters regardless their gender type.

\section{Result}

\subsection{Anthropometry for children and elderly}

This section shows a summary of the anthropometric data of Indonesian children and elderly, both male and female taking into account Chinese and non-Chinese classification (see Tables 1-3). In general, it shows that the male children tend to have the same dimensions as the female children do at both age sub-groups (i.e., 6-9 years and 10-12 years), except for Chinese sub-group at the age 6-9 years. At the 6-9 years sub-group, the Chinese female children tend to have larger dimensions than the Chinese male children. It may be related to the fact that the physical growth of boys, in general, is about 2 years later than that of girls (Gavin, 2015).

Compared to children sample, the anthropometric dimensions of male elderly were larger than those of the female elderly at both Chinese and non-Chinese sub-groups.

\subsection{Comparison of values of stature, weight and BMI for elderly and children samples}

A summary of the basic descriptive statistics of stature and weight (mean, standard deviation, maximum and minimum values) and BMI is shown in Table 4. It includes both Chinese and non-Chinese sub-groups for male and female samples. Body Mass Index (BMI) is proposed to be a health indicator for a particular group, which is defined as the weight (in $\mathrm{kg}$ ) divided by the square of stature (in $\mathrm{m}^{2}$ ). Referring to WHO (2016), all values of BMI are within the normal range of 18.5-24.99, except for the sub-groups of Chinese children of 6-9 years (see Table 4). 
Table 3

Anthropometric data for elderly with subgroups of Chinese and non-Chinese (all dimensions in $\mathrm{cm}$ ).

\begin{tabular}{|c|c|c|c|c|c|c|c|c|c|c|c|c|c|c|c|c|}
\hline \multirow[t]{3}{*}{ Dimension } & \multicolumn{8}{|l|}{ Male } & \multicolumn{8}{|c|}{ Female } \\
\hline & \multicolumn{4}{|c|}{ Chinese } & \multicolumn{4}{|c|}{ non-Chinese } & \multicolumn{4}{|c|}{ Chinese } & \multicolumn{4}{|c|}{ non-Chinese } \\
\hline & 5 th & 50th & 95th & SD & 5 th & 50th & 95th & SD & 5 th & 50th & 95th & SD & 5 th & 50th & 95th & SD \\
\hline 1. Stature & 140.4 & 159.9 & 179.5 & 11.9 & 138.0 & 158.9 & 179.8 & 12.7 & 128.7 & 153.5 & 178.2 & 15.0 & 124.2 & 149.7 & 175.1 & 15.5 \\
\hline 2. Eye height & 128.1 & 148.6 & 169.1 & 12.5 & 126.7 & 148.0 & 169.4 & 13.0 & 114.0 & 139.8 & 165.7 & 15.7 & 113.0 & 137.9 & 162.8 & 15.1 \\
\hline 3. Shoulder height & 112.6 & 130.8 & 149.1 & 11.1 & 111.7 & 130.3 & 148.9 & 11.3 & 91.5 & 114.4 & 137.3 & 13.9 & 91.5 & 113.7 & 135.8 & 13.5 \\
\hline 4. Elbow height & 85.8 & 97.1 & 108.4 & 6.9 & 87.8 & 98.5 & 109.3 & 6.5 & 73.8 & 91.2 & 108.6 & 10.6 & 75.3 & 93.0 & 110.7 & 10.7 \\
\hline 5. Hip height & 77.5 & 89.7 & 101.9 & 7.4 & 78.4 & 90.4 & 102.4 & 7.3 & 66.6 & 84.4 & 102.2 & 10.8 & 64.5 & 82.9 & 101.3 & 11.2 \\
\hline 6. Knuckel height & 57.3 & 67.8 & 78.2 & 6.3 & 58.1 & 68.2 & 78.3 & 6.1 & 49.1 & 63.7 & 78.3 & 8.9 & 50.7 & 63.3 & 75.9 & 7.7 \\
\hline 7. Fingertip height & 49.3 & 59.0 & 68.8 & 5.9 & 49.1 & 58.7 & 68.3 & 5.8 & 41.3 & 55.7 & 70.1 & 8.7 & 42.1 & 55.7 & 69.3 & 8.3 \\
\hline 8. Sitting height & 68.4 & 79.5 & 90.5 & 6.7 & 68.3 & 78.9 & 89.6 & 6.5 & 47.3 & 66.5 & 85.7 & 11.7 & 46.4 & 66.7 & 86.9 & 12.3 \\
\hline 9. Sitting eye height & 54.6 & 65.7 & 76.8 & 6.8 & 55.3 & 65.9 & 76.6 & 6.5 & 43.6 & 59.8 & 76.0 & 9.8 & 42.3 & 59.0 & 75.7 & 10.1 \\
\hline 10. Sitting shoulder height & 43.1 & 54.4 & 65.6 & 6.8 & 42.7 & 53.1 & 63.4 & 6.3 & 35.6 & 48.3 & 61.1 & 7.7 & 35.6 & 48.3 & 61.0 & 7.7 \\
\hline 11. Sitting elbow height & 14.9 & 23.4 & 31.9 & 5.2 & 14.1 & 22.8 & 31.5 & 5.3 & 9.0 & 18.1 & 27.3 & 5.6 & 8.6 & 18.6 & 28.6 & 6.1 \\
\hline 12. Thigh thickness & 10.3 & 15.1 & 19.9 & 2.9 & 9.6 & 14.9 & 20.2 & 3.2 & 6.5 & 12.6 & 18.8 & 3.7 & 6.1 & 12.5 & 19.0 & 3.9 \\
\hline 13. Buttock-knee length & 50.8 & 57.7 & 64.5 & 4.2 & 50.8 & 57.5 & 64.1 & 4.0 & 41.9 & 54.3 & 66.7 & 7.6 & 42.1 & 54.5 & 66.8 & 7.5 \\
\hline 14. Buttock-popliteal length & 41.2 & 48.6 & 55.9 & 4.5 & 41.0 & 48.3 & 55.6 & 4.4 & 36.1 & 45.8 & 55.5 & 5.9 & 36.4 & 45.8 & 55.1 & 5.7 \\
\hline 15. Knee height & 47.3 & 54.8 & 62.3 & 4.6 & 46.9 & 54.5 & 62.1 & 4.6 & 42.8 & 52.9 & 63.0 & 6.1 & 41.8 & 51.9 & 62.0 & 6.1 \\
\hline 16. Popliteal height & 34.3 & 42.2 & 50.1 & 4.8 & 34.0 & 41.6 & 49.1 & 4.6 & 32.4 & 41.9 & 51.3 & 5.7 & 33.5 & 42.1 & 50.7 & 5.2 \\
\hline 17. Shoulder breadth (bideltoid) & 38.2 & 46.9 & 55.6 & 5.3 & 38.0 & 46.4 & 54.8 & 5.1 & 28.3 & 41.2 & 54.2 & 7.9 & 27.1 & 40.6 & 54.0 & 8.2 \\
\hline 18. Shoulder breadth (biacromial) & 28.5 & 35.6 & 42.7 & 4.3 & 28.0 & 35.0 & 41.9 & 4.2 & 22.1 & 33.0 & 43.9 & 6.6 & 22.0 & 33.3 & 44.5 & 6.8 \\
\hline 19. Hip breadth & 34.5 & 45.5 & 56.4 & 6.7 & 34.3 & 44.9 & 55.5 & 6.4 & 30.3 & 43.5 & 56.6 & 8.0 & 30.7 & 43.4 & 56.2 & 7.8 \\
\hline 20. Chest (bust) depth & 15.3 & 25.3 & 35.2 & 6.0 & 15.4 & 25.7 & 36.0 & 6.3 & 11.0 & 22.7 & 34.5 & 7.1 & 11.3 & 23.1 & 35.0 & 7.2 \\
\hline 21. Abdominal depth & 15.9 & 28.6 & 41.3 & 7.7 & 15.7 & 28.4 & 41.1 & 7.7 & 12.0 & 28.1 & 44.2 & 9.8 & 13.3 & 28.6 & 43.8 & 9.3 \\
\hline 22. Shoulder-elbow length & 28.8 & 33.9 & 39.0 & 3.1 & 27.7 & 33.4 & 39.1 & 3.5 & 22.5 & 30.0 & 37.5 & 4.6 & 21.5 & 29.6 & 37.8 & 5.0 \\
\hline 23. Elbow-fingertip length & 37.9 & 44.4 & 50.8 & 3.9 & 38.4 & 44.6 & 50.7 & 3.7 & 27.4 & 38.5 & 49.5 & 6.7 & 28.2 & 38.7 & 49.2 & 6.4 \\
\hline 24. Upper limb length & 60.6 & 70.4 & 80.3 & 6.0 & 61.2 & 71.0 & 80.7 & 5.9 & 55.1 & 65.9 & 76.6 & 6.5 & 55.7 & 67.1 & 78.5 & 6.9 \\
\hline 25. Shoulder-grip length & 49.2 & 54.5 & 59.9 & 3.2 & 49.0 & 54.6 & 60.2 & 3.4 & 39.5 & 49.5 & 59.5 & 6.1 & 40.1 & 49.9 & 59.6 & 5.9 \\
\hline 26. Head length & 14.9 & 18.5 & 22.2 & 2.2 & 14.8 & 18.6 & 22.5 & 2.3 & 12.9 & 18.4 & 23.9 & 3.3 & 12.4 & 17.8 & 23.2 & 3.3 \\
\hline 27. Head breadth & 17.4 & 22.2 & 27.0 & 2.9 & 17.6 & 22.3 & 27.0 & 2.9 & 13.7 & 20.6 & 27.4 & 4.2 & 13.6 & 20.4 & 27.3 & 4.2 \\
\hline 28. Hand length & 17.8 & 20.1 & 22.4 & 1.4 & 17.7 & 19.9 & 22.2 & 1.3 & 11.0 & 17.6 & 24.3 & 4.0 & 11.1 & 17.2 & 23.4 & 3.8 \\
\hline 29. Hand breadth & 7.9 & 10.4 & 13.0 & 1.6 & 7.5 & 10.2 & 12.9 & 1.7 & 6.8 & 10.6 & 14.4 & 2.3 & 6.8 & 10.5 & 14.2 & 2.3 \\
\hline 30. Foot length & 20.8 & 24.8 & 28.7 & 2.4 & 20.8 & 24.9 & 29.0 & 2.5 & 16.8 & 22.3 & 27.8 & 3.4 & 17.2 & 23.0 & 28.8 & 3.5 \\
\hline 31. Foot breadth & 8.7 & 11.3 & 13.9 & 1.6 & 8.8 & 11.5 & 14.1 & 1.6 & 7.6 & 10.7 & 13.7 & 1.9 & 7.6 & 11.0 & 14.3 & 2.0 \\
\hline 32. Span & 129.9 & 154.3 & 178.8 & 14.9 & 128.7 & 153.2 & 177.8 & 14.9 & 117.1 & 143.9 & 170.7 & 16.3 & 116.3 & 144.1 & 171.9 & 16.9 \\
\hline 33. Elbow span & 63.1 & 75.7 & 88.3 & 7.7 & 63.4 & 75.4 & 87.5 & 7.3 & 59.3 & 72.8 & 86.3 & 8.2 & 60.3 & 72.8 & 85.4 & 7.6 \\
\hline 34. Vertical grip reach (standing) & 158.1 & 193.4 & 228.6 & 21.4 & 159.0 & 193.5 & 227.9 & 20.9 & 151.6 & 191.8 & 232.0 & 24.4 & 141.7 & 184.4 & 227.1 & 26.0 \\
\hline 35. Vertical grip reach (sitting) & 105.1 & 131.6 & 158.1 & 16.1 & 105.7 & 130.4 & 155.2 & 15.1 & 88.3 & 123.0 & 157.7 & 21.1 & 87.0 & 123.3 & 159.5 & 22.0 \\
\hline 36. Forward grip reach & 57.0 & 65.6 & 74.2 & 5.2 & 55.7 & 65.1 & 74.5 & 5.7 & 52.1 & 63.9 & 75.7 & 7.2 & 53.6 & 64.4 & 75.3 & 6.6 \\
\hline
\end{tabular}

Table 4

Statistic descriptive of stature and weight dimensions.

\begin{tabular}{|c|c|c|c|c|c|c|c|c|c|}
\hline \multirow[t]{2}{*}{ Group } & \multicolumn{4}{|c|}{ Stature (in cm) } & \multicolumn{4}{|c|}{ Weight (in kg) } & \multirow[t]{2}{*}{$\mathrm{BMI}^{\mathrm{a}}$} \\
\hline & Mean & SD & Max & Min & Mean & SD & $\operatorname{Max}$ & Min & \\
\hline \multicolumn{10}{|l|}{ Children ( $6-9$ years) } \\
\hline Male Chinese & 117.8 & 10.4 & 153 & 100 & 22.3 & 8.3 & 53 & 15 & 16.1 \\
\hline Female Chinese & 121.9 & 8.4 & 143.5 & 97 & 26.7 & 7.1 & 47 & 17 & 17.9 \\
\hline Male non-Chinese & 121.7 & 10.4 & 141 & 92 & 29.5 & 9.2 & 64 & 15 & 19.9 \\
\hline Female non-Chinese & 122.8 & 20.3 & 162 & 97 & 30.9 & 8.9 & 55 & 18 & 20.5 \\
\hline \multicolumn{10}{|c|}{ Children (10-12 years) } \\
\hline Male Chinese & 139.1 & 9.2 & 155 & 116 & 36.9 & 8.9 & 55 & 21 & 19.1 \\
\hline Female Chinese & 140.9 & 10.2 & 163 & 110 & 37.6 & 7.8 & 61 & 20 & 18.9 \\
\hline Male non-Chinese & 140.3 & 9.9 & 163 & 107 & 40.6 & 11.3 & 74 & 15 & 20.6 \\
\hline Female non-Chinese & 137 & 15.5 & 163 & 100 & 36.3 & 12.3 & 61 & 17 & 19.4 \\
\hline \multicolumn{10}{|l|}{ Elderly } \\
\hline Male Chinese & 159.9 & 11.9 & 179 & 137 & 58.8 & 11.3 & 79 & 40 & 22.9 \\
\hline Female Chinese & 153.5 & 15.4 & 178 & 125 & 52.1 & 15.3 & 78 & 25 & 22.1 \\
\hline Male non-Chinese & 158.9 & 12.7 & 179 & 137 & 58.5 & 11.3 & 79 & 40 & 23.2 \\
\hline Female non-Chinese & 149.7 & 15.5 & 178 & 124 & 50.3 & 14.6 & 78 & 25 & 22.4 \\
\hline
\end{tabular}

a Body Mass Index (in $\mathrm{kg} / \mathrm{m}^{2}$ ) = weight (in $\mathrm{kg}$ )/squared-stature (in $\mathrm{m}^{2}$ ).

\subsection{Comparison of the 50th percentile mean values for Chinese and non-Chinese samples}

A comparison of the 36 dimensions measured between Chinese and non-Chinese sub-group for both male and female samples in both children and elderly population groups, as shown in Tables 5 and 6. The Student's t-test for independent mean difference was used. It shows that more than 50 percent of the dimensions have been significantly different, at the male and female children of 6-9 years sub-group. The non-Chinese tends to be higher, wider, and bigger than its counterpart. At the children of 10-12 years subgroup at both male and female, it seems that there is no significant difference between Chinese and non-Chinese sub-group.

More interestingly, comparing the Chinese and non-Chinese 
Table 5

Comparison of the 50th percentile values for Chinese and non-Chinese for children population (all dimensions in $\mathrm{cm}$ ).

\begin{tabular}{|c|c|c|c|c|c|c|c|c|c|c|c|c|c|c|c|c|c|c|c|c|}
\hline \multirow[t]{4}{*}{ Dimension } & \multicolumn{10}{|c|}{ Children (6-9 years) } & \multicolumn{10}{|c|}{ Children (10-12 years) } \\
\hline & \multicolumn{5}{|l|}{ Male } & \multicolumn{5}{|c|}{ Female } & \multicolumn{5}{|l|}{ Male } & \multicolumn{5}{|c|}{ Female } \\
\hline & \multicolumn{2}{|c|}{ Chinese } & \multicolumn{2}{|c|}{ non-Chinese } & \multirow[t]{2}{*}{$p$} & \multicolumn{2}{|c|}{ Chinese } & \multicolumn{2}{|c|}{ non-Chinese } & \multirow[t]{2}{*}{$p$} & \multicolumn{2}{|c|}{ Chinese } & \multicolumn{2}{|c|}{ non-Chinese } & \multirow[t]{2}{*}{$p$} & \multicolumn{2}{|c|}{ Chinese } & \multicolumn{2}{|c|}{ non-Chinese } & \multirow[t]{2}{*}{$p$} \\
\hline & 50th & SD & 50th & SD & & 50th & SD & 50th & SD & & 50th & SD & 50th & SD & & 50th & SD & 50 th & SD & \\
\hline 1. Stature & 117.8 & 10.4 & 121.7 & 10.4 & 0.02 & 121.9 & 8.4 & 122.8 & 20.3 & 0.39 & 139.1 & 9.2 & 140.3 & 10.0 & 0.31 & 141.0 & 10.2 & 137.0 & 15.5 & 0.97 \\
\hline 2. Eye height & 101.2 & 12.5 & 111.3 & 11.1 & 0.00 & 110.0 & 8.1 & 116.4 & 16.5 & 0.02 & 126.9 & 8.3 & 126.7 & 10.4 & 0.78 & 130.7 & 10.9 & 121.1 & 18.8 & 0.99 \\
\hline 3. Shoulder height & 90.2 & 10.8 & 99.3 & 12.2 & 0.00 & 97.6 & 8.8 & 101.1 & 15.7 & 0.11 & 112.3 & 8.4 & 112.4 & 12.3 & 0.48 & 113.6 & 9.3 & 104.0 & 16.7 & 0.99 \\
\hline 4. Elbow height & 68.1 & 8.2 & 76.6 & 8.7 & 0.00 & 74.7 & 5.5 & 84.6 & 14.8 & 0.00 & 87.0 & 7.0 & 84.7 & 11.0 & 0.88 & 87.2 & 7.2 & 85.0 & 14.3 & 0.89 \\
\hline 5. Hip height & 59.2 & 9.5 & 67.8 & 10.2 & 0.00 & 67.0 & 6.3 & 74.1 & 15.1 & 0.00 & 76.7 & 7.1 & 75.8 & 9.3 & 0.68 & 79.9 & 8.2 & 75.6 & 15.6 & 0.99 \\
\hline 6. Knuckel height & 46.1 & 6.5 & 51.0 & 6.4 & 0.00 & 50.8 & 4.7 & 57.6 & 8.9 & 0.00 & 59.5 & 6.5 & 58.4 & 8.4 & 0.48 & 60.1 & 6.0 & 56.2 & 9.1 & 0.99 \\
\hline 7. Fingertip height & 39.3 & 5.9 & 44.4 & 5.8 & 0.00 & 43.8 & 3.0 & 51.7 & 9.0 & 0.00 & 50.7 & 5.3 & 48.5 & 6.3 & 0.37 & 51.3 & 5.3 & 50.3 & 8.0 & 0.83 \\
\hline 8. Sitting height & 61.0 & 5.2 & 64.9 & 6.4 & 0.01 & 63.0 & 4.7 & 67.0 & 8.6 & 0.01 & 70.6 & 5.1 & 68.0 & 6.6 & 0.98 & 70.9 & 5.8 & 67.5 & 9.1 & 0.99 \\
\hline 9. Sitting eye height & 52.1 & 7.2 & 53.4 & 7.1 & 0.20 & 54.0 & 4.1 & 59.0 & 9.4 & 0.00 & 60.1 & 5.3 & 58.6 & 7.6 & 0.77 & 60.6 & 6.4 & 62.3 & 9.7 & 0.09 \\
\hline 10. Sitting shoulder height & 7.2 & 6.6 & 41.9 & 7.3 & 0.00 & 41.1 & 4.0 & 43.5 & 8.6 & 0.03 & 46.9 & 5.5 & 45.5 & 7.0 & 0.79 & 45.7 & 5.8 & 42.6 & 9.6 & 0.99 \\
\hline 11. Sitting elbow height & 14.9 & 3.2 & 15.5 & 4.6 & 0.19 & 16.8 & 3.7 & 17.8 & 6.1 & 0.18 & 18.4 & 3.9 & 17.5 & 4.6 & 0.85 & 18.1 & 4.3 & 18.0 & 6.1 & 0.55 \\
\hline 12. Thigh thickness & 10.3 & 2.8 & 9.9 & 2.8 & 0.79 & 8.8 & 1.9 & 9.9 & 2.8 & 0.02 & 10.7 & 2.6 & 11.8 & 3.9 & 0.31 & 10.9 & 2.3 & 10.7 & 2.9 & 0.69 \\
\hline 13. Buttock-knee length & 35.2 & 6.0 & 40.2 & 6.2 & 0.00 & 40.0 & 3.2 & 43.7 & 8.1 & 0.01 & 46.0 & 4.3 & 44.4 & 6.5 & 0.92 & 46.6 & 5.3 & 42.9 & 8.8 & 0.99 \\
\hline 14. Buttock-popliteal length & 29.5 & 5.5 & 34.5 & 5.5 & 0.00 & 34.2 & 3.2 & 38.3 & 6.9 & 0.00 & 39.3 & 5.1 & 37.5 & 5.9 & 0.94 & 39.6 & 4.1 & 37.7 & 6.2 & 0.99 \\
\hline 15. Knee height & 37.7 & 5.7 & 40.2 & 7.5 & 0.05 & 42.2 & 6.3 & 44.4 & 9.3 & 0.10 & 44.8 & 4.4 & 42.2 & 5.0 & 0.99 & 44.4 & 4.8 & 45.1 & 9.0 & 0.26 \\
\hline 16. Popliteal height & 31.4 & 4.2 & 33.1 & 4.7 & 0.01 & 32.7 & 4.3 & 36.7 & 6.9 & 0.00 & 37.5 & 3.2 & 36.8 & 5.0 & 0.79 & 37.5 & 4.6 & 36.1 & 6.5 & 0.94 \\
\hline 17. Shoulder breadth (bideltoid) & 29.9 & 3.4 & 32.1 & 4.6 & 0.00 & 31.2 & 3.5 & 35.9 & 9.4 & 0.00 & 34.4 & 4.9 & 33.8 & 6.2 & 0.52 & 35.5 & 6.1 & 35.4 & 8.4 & 0.53 \\
\hline 18. Shoulder breadth (biacromial) & 22.7 & 4.8 & 25.7 & 7.0 & 0.00 & 24.3 & 5.0 & 28.4 & 9.0 & 0.00 & 28.5 & 4.9 & 27.8 & 6.8 & 0.73 & 29.2 & 6.8 & 26.6 & 8.2 & 0.98 \\
\hline 19. Hip breadth & 24.1 & 3.9 & 26.5 & 5.9 & 0.01 & 26.0 & 3.9 & 29.3 & 9.0 & 0.02 & 31.1 & 5.5 & 27.8 & 5.2 & 0.99 & 29.8 & 5.9 & 30.5 & 8.5 & 0.27 \\
\hline 20. Chest (bust) depth & 14.6 & 2.6 & 14.5 & 3.9 & 0.57 & 15.1 & 2.6 & 15.8 & 5.7 & 0.24 & 17.0 & 3.1 & 16.5 & 3.5 & 0.76 & 16.1 & 3.5 & 17.1 & 4.9 & 0.58 \\
\hline 21. Abdominal depth & 16.0 & 3.2 & 17.4 & 4.8 & 0.02 & 16.7 & 2.5 & 17.5 & 6.3 & 0.23 & 17.8 & 4.5 & 19.2 & 4.5 & 0.07 & 16.9 & 4.6 & 16.8 & 6.4 & 0.61 \\
\hline 22. Shoulder-elbo & 22.5 & 3.8 & 25.2 & 6.6 & 0.02 & 24.6 & 3.9 & 25.0 & 8.7 & 0.40 & 29.0 & 4.0 & 28.6 & 6.2 & 0.65 & 29.0 & 4.0 & 26.3 & 8.0 & 0.99 \\
\hline 23. Elbow-fingertip length & 30.0 & 4.2 & 33.6 & 5.1 & 0.03 & 31.8 & 2.8 & 35.9 & 11.4 & 0.02 & 37.7 & 3.9 & 37.4 & 4.4 & 0.51 & 38.8 & 4.6 & 34.0 & 9.6 & 0.99 \\
\hline 24. Upper limb length & 48.3 & 7.9 & 52.1 & 7.0 & 0.00 & 50.7 & 5.1 & 52.8 & 11.1 & 0.14 & 60.8 & 4.5 & 59.9 & 7.4 & 0.67 & 61.3 & 6.4 & 51.7 & 10.1 & 0.99 \\
\hline 25. Shoulder-grip length & 37.7 & 6.9 & 43.3 & 6.8 & 0.00 & 42.3 & 4.5 & 48.7 & 10.3 & 0.00 & 48.6 & 5.4 & 50.7 & 7.6 & 0.06 & 51.6 & 5.9 & 47.1 & 10.6 & 0.99 \\
\hline 26. Head length & 16.3 & 2.7 & 17.8 & 3.1 & 0.00 & 16.7 & 1.7 & 18.6 & 2.8 & 0.00 & 18.3 & 2.6 & 18.2 & 3.3 & 0.56 & 17.7 & 2.4 & 18.2 & 3.2 & 0.13 \\
\hline 27. Head breadth & 15.8 & 3.8 & 16.8 & 4.3 & 0.04 & 17.2 & 3.2 & 19.2 & 6.8 & 0.05 & 17.4 & 2.5 & 17.2 & 4.5 & 0.74 & 18.5 & 4.4 & 18.8 & 5.9 & 0.36 \\
\hline 28. Hand length & 12.4 & 1.7 & 13.9 & 2.0 & 0.00 & 14.2 & 1.7 & 15.8 & 3.3 & 0.00 & 15.7 & 1.7 & 15.2 & 2.4 & 0.59 & 16.1 & 2.2 & 14.7 & 3.2 & 0.99 \\
\hline 29. Hand breadth & 6.1 & 0.8 & 6.6 & 1.1 & 0.00 & 6.6 & 0.8 & 6.2 & 1.8 & 0.89 & 7.2 & 0.9 & 7.0 & 1.2 & 0.82 & 7.2 & 1.3 & 7.1 & 2.0 & 0.65 \\
\hline 30. Foot length & 17.1 & 2.5 & 19.3 & 2.8 & 0.00 & 19.3 & 2.2 & 19.4 & 3.1 & 0.43 & 21.7 & 1.9 & 21.9 & 3.8 & 0.77 & 21.2 & 1.8 & 20.3 & 3.6 & 0.98 \\
\hline 31. Foot breadth & 6.8 & 1.5 & 7.7 & 1.4 & 0.00 & 7.7 & 1.2 & 8.5 & 2.4 & 0.03 & 8.9 & 1.3 & 8.4 & 1.3 & 0.96 & 8.6 & 1.4 & 8.5 & 2.2 & 0.64 \\
\hline 32. Span & 108.2 & 14.0 & 122.2 & 13.7 & 0.00 & 118.0 & 11.0 & 130.3 & 21.2 & 0.00 & 137.5 & 8.8 & 136.3 & 13.9 & 0.69 & 141.4 & 11.1 & 129.3 & 22.0 & 0.99 \\
\hline 33. Elbow span & 61.8 & 11.8 & 63.4 & 9.5 & 0.00 & 61.7 & 5.7 & 67.7 & 12.6 & $\mathbf{0 . 0 0}$ & 70.0 & 6.2 & 70.4 & 9.7 & 0.40 & 72.8 & 7.0 & 68.0 & 11.5 & 0.99 \\
\hline 34. Vertical grip reach (standing) & 132.1 & 15.2 & 146.1 & 16.9 & 0.00 & 143.4 & 11.8 & 160.0 & 24.8 & 0.00 & 164.0 & 9.4 & 166.7 & 17.4 & 0.17 & 167.5 & 14.4 & 156.0 & 22.6 & 0.99 \\
\hline 35. Vertical grip reach (sitting) & 83.9 & 12.5 & 86.6 & 10.6 & 0.00 & 81.8 & 7.1 & 86.9 & 16.4 & 0.02 & 94.1 & 7.1 & 93.4 & 10.6 & 0.65 & 96.1 & 9.7 & 85.7 & 15.7 & 0.99 \\
\hline 36. Forward grip reach & 45.3 & 8.6 & 51.6 & 9.0 & 0.00 & 49.9 & 7.1 & 59.8 & 13.2 & 0.00 & 56.4 & 6.6 & 57.3 & 8.5 & 0.53 & 59.9 & 7.5 & 59.0 & 13.3 & 0.59 \\
\hline
\end{tabular}

Notes: bolded figures show significant difference in means at $\alpha \leq 5 \%$.

elderly sub-group, similar to the children of 10-12 years group, there is no significant difference, at both male and female group.

\subsection{Empirical prediction of anthropometric dimensions using Drilis and Contini approach}

According to Peacock et al. (2012) and Hartono and Gunawan (2015), particular body dimensions are significantly associated either with stature or body weight. Before the body dimensions can be used as a measure of prediction, it is necessary to assess their validity and reliability in Indonesian special population context. For content validity testing, it refers to the extent to which a measure represents meanings of a given concept (Babbie, 1992). Here, the instrument of 36 body dimensions were adopted from Pheasant and Haslegrave (2006) and Chuan et al. (2010). Their thoroughness suggests that 36 body dimensions do measure and represent the anthropometry of certain population. For reliability testing, the measure was assessed using Cronbach's Alpha. The cut-off value was set to 0.7. The result of two special population data was deemed satisfactory (as shown in Table 7). The data from two populations (i.e., children and elderly) were then analyzed independently to determine the number of factors to extract. Methods used were principal components with varimax rotation (Pitt et al., 1995). According to factor analysis, at the children group, it indicated that there were two major factors extracted, and these explained about $62 \%$ of the variance. The first factor included the dimensions of numbers $1,2,3,4,5,6,7,8,9,10,13,14,16,23,24,32$, $33,34,35$, and 36 , which were related to limb segment (please see Fig. 1 for the description of body dimension numbers). The second factor included the dimensions of numbers 25, 28, 29, 30, and 31, those were related to girth and width segment. Similar to the children group, there were two factors which explained $60 \%$ of the variance for the elderly group. The first factor included the dimensions 1 to $11,13,14,17,18,22,23,24,25,28,30,32,33,34,35$ and 36 , which were related to limb segment, while the second factors consisted of dimensions 12, 15, 19, 20, 21, 26, 27, 29 and 31. This finding confirmed the generic model of the relationship between limb segment and stature, and girth/width segment and weight, respectively (see Peacock et al., 2012; Hartono, 2016).

By using correlation analysis, it was found that the stature was highly correlated with limb lengths, whilst the body weight was found to be a good predictor of width or girth measures (Hartono and Gunawan, 2015). Tables 8 and 9 show the significant correlations (with significant value of 0.05 ) and ratios of common body measures with stature and weight for both male and female samples, applies to children (at both 6-9 and 10-12 years) and elderly groups for both Chinese and non-Chinese sub-group.

The common significant body dimensions associated with stature were eye height, shoulder height, elbow height, hip height, fingertip height, sitting eye height, span, and vertical grip reach 
Table 6

Comparison of the 50th percentile values for Chinese and non-Chinese for elderly population (all dimensions in $\mathrm{cm}$ ).

\begin{tabular}{|c|c|c|c|c|c|c|c|c|c|c|}
\hline \multirow[t]{4}{*}{ Dimension } & \multicolumn{10}{|c|}{ Elderly } \\
\hline & \multicolumn{5}{|l|}{ Male } & \multicolumn{5}{|c|}{ Female } \\
\hline & \multicolumn{2}{|c|}{ Chinese } & \multicolumn{2}{|c|}{ non-Chinese } & \multirow[t]{2}{*}{$p$} & \multicolumn{2}{|c|}{ Chinese } & \multicolumn{2}{|c|}{ non-Chinese } & \multirow[t]{2}{*}{$p$} \\
\hline & 50th & SD & 50th & SD & & 50th & SD & 50th & SD & \\
\hline 1. Stature & 159.9 & 11.9 & 158.9 & 12.7 & 0.47 & 153.5 & 15.0 & 149.7 & 15.5 & 0.04 \\
\hline 2. Eye height & 148.6 & 12.5 & 148.0 & 13.0 & 0.65 & 139.8 & 15.7 & 137.9 & 15.1 & 0.22 \\
\hline 3. Shoulder height & 130.8 & 11.1 & 130.3 & 11.3 & 0.64 & 114.4 & 13.9 & 113.7 & 13.5 & 0.45 \\
\hline 4. Elbow height & 97.1 & 6.9 & 98.5 & 6.5 & 0.48 & 91.2 & 10.6 & 93.0 & 10.7 & 0.18 \\
\hline 5. Hip height & 89.7 & 7.4 & 90.4 & 7.3 & 0.43 & 84.4 & 10.8 & 82.9 & 11.2 & 0.12 \\
\hline 6. Knuckel height & 67.8 & 6.3 & 68.2 & 6.1 & 0.45 & 63.7 & 8.9 & 63.3 & 7.7 & 0.56 \\
\hline 7. Fingertip height & 59.0 & 5.9 & 58.7 & 5.8 & 0.45 & 55.7 & 8.7 & 55.7 & 8.3 & 0.81 \\
\hline 8. Sitting height & 79.5 & 6.7 & 78.9 & 6.5 & 0.56 & 66.5 & 11.7 & 66.7 & 12.3 & 0.58 \\
\hline 9. Sitting eye height & 65.7 & 6.8 & 65.9 & 6.5 & 0.64 & 59.8 & 9.8 & 59.0 & 10.1 & 0.62 \\
\hline 10. Sitting shoulder height & 54.4 & 6.8 & 53.1 & 6.3 & 0.44 & 48.3 & 7.7 & 48.4 & 7.7 & 0.91 \\
\hline 11. Sitting elbow height & 23.4 & 5.2 & 22.8 & 5.3 & 0.45 & 18.1 & 5.6 & 18.6 & 6.1 & 0.51 \\
\hline 12. Thigh thickness & 15.1 & 2.9 & 14.9 & 3.2 & 0.51 & 12.6 & 3.7 & 12.5 & 3.9 & 0.76 \\
\hline 13. Buttock-knee length & 57.7 & 4.2 & 57.5 & 4.0 & 0.81 & 54.3 & 7.6 & 54.5 & 7.5 & 0.78 \\
\hline 14. Buttock-popliteal length & 48.6 & 4.5 & 48.3 & 4.4 & 0.84 & 45.8 & 5.9 & 45.8 & 5.7 & 0.89 \\
\hline 15. Knee height & 54.8 & 4.6 & 54.5 & 4.6 & 0.56 & 52.9 & 6.1 & 51.9 & 6.1 & 0.14 \\
\hline 16. Popliteal height & 42.2 & 4.8 & 41.6 & 4.6 & 0.42 & 41.9 & 5.7 & 42.1 & 5.2 & 0.23 \\
\hline 17. Shoulder breadth (bideltoid) & 46.9 & 5.3 & 46.4 & 5.1 & 0.51 & 41.2 & 7.9 & 40.6 & 8.2 & 0.18 \\
\hline 18. Shoulder breadth (biacromial) & 35.6 & 4.3 & 35.0 & 4.2 & 0.52 & 33.0 & 6.6 & 33.3 & 6.8 & 0.58 \\
\hline 19. Hip breadth & 45.5 & 6.7 & 44.9 & 6.4 & 0.44 & 43.5 & 8.0 & 43.4 & 7.8 & 0.61 \\
\hline 20. Chest (bust) depth & 25.3 & 6.0 & 25.7 & 6.3 & 0.51 & 22.7 & 7.1 & 23.1 & 7.2 & 0.59 \\
\hline 21. Abdominal depth & 28.6 & 7.7 & 28.4 & 7.7 & 0.62 & 28.1 & 9.8 & 28.6 & 9.3 & 0.64 \\
\hline 22. Shoulder-elbow length & 33.9 & 3.1 & 33.4 & 3.5 & 0.48 & 30.0 & 4.6 & 29.6 & 5.0 & 0.24 \\
\hline 23. Elbow-fingertip length & 44.4 & 3.9 & 44.6 & 3.7 & 0.51 & 38.5 & 6.7 & 38.7 & 6.4 & 0.56 \\
\hline 24. Upper limb length & 70.4 & 6.0 & 71.0 & 5.9 & 0.39 & 65.9 & 6.5 & 67.1 & 6.9 & 0.16 \\
\hline 25. Shoulder-grip length & 54.5 & 3.2 & 54.6 & 3.4 & 0.61 & 49.5 & 6.1 & 49.9 & 5.9 & 0.54 \\
\hline 26. Head length & 18.5 & 2.2 & 18.6 & 2.3 & 0.58 & 18.4 & 3.3 & 17.8 & 3.3 & 0.43 \\
\hline 27. Head breadth & 22.2 & 2.9 & 22.3 & 2.9 & 0.75 & 20.6 & 4.2 & 20.4 & 4.2 & 0.78 \\
\hline 28. Hand length & 20.1 & 1.4 & 19.9 & 1.3 & 0.56 & 17.6 & 4.0 & 17.2 & 3.8 & 0.68 \\
\hline 29. Hand breadth & 10.4 & 1.6 & 10.2 & 1.7 & 0.74 & 10.6 & 2.3 & 10.5 & 2.3 & 0.78 \\
\hline 30. Foot length & 24.8 & 2.4 & 24.9 & 2.5 & 0.76 & 22.3 & 3.4 & 23.0 & 3.5 & 0.41 \\
\hline 31. Foot breadth & 11.3 & 1.6 & 11.5 & 1.6 & 0.71 & 10.7 & 1.9 & 11.0 & 2.0 & 0.38 \\
\hline 32. Span & 154.3 & 14.9 & 153.2 & 14.9 & 0.62 & 143.9 & 16.3 & 144.1 & 16.9 & 0.57 \\
\hline 33. Elbow span & 75.7 & 7.7 & 75.4 & 7.3 & 0.68 & 72.8 & 8.2 & 72.8 & 7.6 & 0.79 \\
\hline 34. Vertical grip reach (standing) & 193.4 & 21.4 & 193.5 & 20.9 & 0.54 & 191.8 & 24.4 & 184.4 & 26.0 & 0.01 \\
\hline 35. Vertical grip reach (sitting) & 131.6 & 16.1 & 130.4 & 15.1 & 0.48 & 123.0 & 21.1 & 123.3 & 22.0 & 0.91 \\
\hline 36. Forward grip reach & 65.6 & 5.2 & 65.1 & 5.7 & 0.51 & 63.9 & 7.2 & 64.4 & 6.6 & 0.41 \\
\hline
\end{tabular}

Notes: bolded figures show significant difference in means at $\alpha \leq 5 \%$.

Table 7

Reliability test for children and elderly anthropometric measures.

\begin{tabular}{|c|c|c|c|}
\hline \multicolumn{2}{|l|}{ Children } & \multicolumn{2}{|l|}{ Elderly } \\
\hline Cronbach's Alpha & Cronbach's Alpha based on Standardized Items & Cronbach's Alpha & Cronbach's Alpha based on Standardized Items \\
\hline 0.928 & 0.934 & 0.961 & 0.972 \\
\hline
\end{tabular}

(standing). At all samples, eye height and shoulder height were found to be highly correlated with stature. It means these two measures can be confidently predicted by known stature. Scale ratio takes place to perform the predicted measures (Pheasant and Haslegrave, 2006; Peacock et al., 2012). Related to body weight, all samples show that thigh thickness and abdominal depth were deemed to be significant measures to be associated with.

\subsection{Comparison of selected new and old anthropometric data based on significant correlation}

The new anthropometric data taken from children and elderly population group were compared, in terms of weight and stature ratio, to the previous data. The previous anthropometric data have been adopted from Chuan et al. (2010) and Hartono and Gunawan
(2015). With respect to the significant correlations between measures as shown in Table 10 and the available published data, there were seven body dimensions taken, i.e., eye height, shoulder height, hip height, fingertip height, sitting eye height, thigh thickness, and abdominal height. Among those five data sets, they tend to have the same ratio values, except for the children data of 6-9 years in the section of weight ratio both Chinese and non-Chinese. Both Chinese and non-Chinese 6-9 years children have higher weight ratio. It may be due to the growing years from birth to early adulthood period, in which children tend to have higher rate for limb segments compared to other body dimensions. According to Kroemer (2006), during the middle childhood (6-12 years), there will be more on horizontal growth and gradual changes in physical appearance. 
Table 8

Significant correlations and ratios with stature and weight for male and female subjects for children.

\begin{tabular}{|c|c|c|c|c|c|c|c|c|c|c|c|c|c|c|c|c|c|c|c|c|}
\hline \multirow[t]{3}{*}{ Dimension } & \multicolumn{10}{|c|}{ Children (6-9 years) } & \multicolumn{10}{|c|}{ Children (10-12 years) } \\
\hline & \multicolumn{2}{|c|}{ p-value } & \multicolumn{2}{|c|}{ W_Cor } & \multicolumn{2}{|c|}{ S_Cor } & \multicolumn{2}{|c|}{ W_Rat } & \multicolumn{2}{|c|}{ S_Rat } & \multicolumn{2}{|c|}{ p-value } & \multicolumn{2}{|c|}{ W_Cor } & \multicolumn{2}{|c|}{ S_Cor } & \multicolumn{2}{|c|}{ W_Rat } & \multicolumn{2}{|c|}{ S_Rat } \\
\hline & C & NC & C & NC & $\mathrm{C}$ & NC & C & NC & C & NC & C & NC & $\mathrm{C}$ & NC & $\mathrm{C}$ & NC & C & NC & C & NC \\
\hline \multicolumn{21}{|l|}{ Male } \\
\hline Eye height & 0 & 0 & 0.56 & 0.68 & 0.98 & 0.81 & 4.55 & 3.77 & 0.86 & 0.91 & 0 & 0 & 0.27 & 0.25 & 0.94 & 0.91 & 3.43 & 3.12 & 0.91 & 0.9 \\
\hline Shoulder height & 0 & 0 & 0.77 & 0.81 & 0.93 & 0.79 & 4.05 & 3.37 & 0.77 & 0.82 & 0 & 0 & 0.3 & 0.4 & 0.94 & 0.95 & 3.04 & 2.76 & 0.81 & 0.8 \\
\hline Elbow height & 0 & 0 & 0.75 & 0.75 & 0.91 & 0.97 & 3.06 & 2.60 & 0.58 & 0.63 & 0 & 0 & 0.34 & 0.43 & 0.87 & 0.83 & 2.36 & 2.08 & 0.63 & 0.6 \\
\hline Hip height & 0 & 0 & 0.77 & 0.78 & 0.89 & 0.76 & 2.66 & 2.30 & 0.5 & 0.56 & 0 & 0 & 0.35 & 0.43 & 0.82 & 0.78 & 2.08 & 1.87 & 0.55 & 0.54 \\
\hline Fingertip height & 0 & 0 & 0.69 & 0.67 & 0.86 & 0.9 & 1.77 & 1.50 & 0.33 & 0.36 & 0.01 & 0.02 & 0.21 & 0.45 & 0.78 & 0.91 & 1.37 & 1.19 & 0.36 & 0.35 \\
\hline Sitting eye height & 0 & 0 & 0.56 & 0.6 & 0.87 & 0.85 & 2.34 & 1.81 & 0.44 & 0.44 & 0 & 0 & 0.25 & 0.44 & 0.84 & 0.72 & 1.63 & 1.44 & 0.43 & 0.42 \\
\hline Span & .02 & 0 & 0.34 & 0.39 & 0.68 & 0.9 & 4.86 & 4.14 & 0.92 & 1 & 0 & 0 & 0.29 & 0.31 & 0.69 & 0.64 & 3.72 & 3.35 & 0.99 & 0.97 \\
\hline Vertical grip reach (standing) & 0 & 0 & 0.59 & 0.67 & 0.79 & 0.81 & 5.94 & 4.95 & 1.12 & 1.2 & 0 & 0 & 0.39 & 0.34 & 0.71 & 0.72 & 4.44 & 4.10 & 1.18 & 1.19 \\
\hline Thigh thickness & 0 & 0 & 0.94 & 0.83 & 0.77 & 0.77 & 0.46 & 0.34 & 0.09 & 0.08 & 0 & 0 & 0.71 & 0.68 & 0.45 & 0.34 & 0.29 & 0.29 & 0.08 & 0.08 \\
\hline Abdominal depth & 0 & 0 & 0.91 & 0.8 & 0.75 & 0.81 & 0.72 & 0.59 & 0.14 & 0.12 & 0 & 0 & 0.73 & 0.66 & 0.14 & 0.36 & 0.48 & 0.47 & 0.13 & 0.14 \\
\hline \multicolumn{21}{|l|}{ Female } \\
\hline Eye height & 0 & 0 & 0.67 & 0.73 & 0.96 & 0.91 & 4.12 & 3.76 & 0.9 & 0.95 & 0 & 0 & 0.55 & 0.3 & 0.94 & 0.94 & 3.48 & 3.33 & 0.93 & 0.88 \\
\hline Shoulder height & .01 & 0.01 & 0.52 & 0.66 & 0.86 & 0.9 & 3.66 & 3.27 & 0.8 & 0.82 & 0 & 0 & 0.54 & 0.51 & 0.91 & 0.88 & 3.02 & 2.86 & 0.81 & 0.76 \\
\hline Elbow height & 0 & 0 & 0.7 & 0.74 & 0.85 & 0.95 & 2.80 & 2.74 & 0.61 & 0.69 & 0 & 0 & 0.42 & 0.43 & 0.58 & 0.57 & 2.32 & 2.34 & 0.62 & 0.62 \\
\hline Hip height & 0 & 0 & 0.63 & 0.68 & 0.92 & 0.88 & 2.51 & 2.40 & 0.55 & 0.6 & 0 & 0 & 0.47 & 0.39 & 0.67 & 0.78 & 2.12 & 2.08 & 0.57 & 0.55 \\
\hline Fingertip height & 0 & 0 & 0.71 & 0.52 & 0.93 & 0.88 & 1.64 & 1.67 & 0.36 & 0.42 & 0.01 & 0 & 0.49 & 0.37 & 0.77 & 0.48 & 1.36 & 1.39 & 0.36 & 0.37 \\
\hline Sitting eye height & 0 & 0 & 0.67 & 0.74 & 0.86 & 0.92 & 2.02 & 1.91 & 0.44 & 0.48 & 0 & 0.02 & 0.31 & 0.42 & 0.65 & 0.68 & 1.61 & 1.71 & 0.43 & 0.45 \\
\hline Span & 0 & 0 & 0.49 & 0.73 & 0.77 & 0.78 & 4.42 & 4.21 & 0.97 & 1.06 & 0 & 0 & 0.39 & 0.39 & 0.81 & 0.84 & 3.76 & 3.56 & 1 & 0.94 \\
\hline Vertical grip reach (standing) & 0 & 0 & 0.43 & 0.66 & 0.76 & 0.79 & 5.37 & 5.17 & 1.18 & 1.3 & 0 & 0 & 0.28 & 0.32 & 0.76 & 0.75 & 4.45 & 4.29 & 1.19 & 1.14 \\
\hline Thigh thickness & 0 & 0 & 0.81 & 0.79 & 0.81 & 0.64 & 0.33 & 0.32 & 0.07 & 0.08 & 0 & 0 & 0.81 & 0.62 & 0.44 & 0.03 & 0.29 & 0.29 & 0.08 & 0.08 \\
\hline Abdominal depth & 0 & 0 & 0.78 & 0.78 & 0.73 & 0.53 & 0.62 & 0.56 & 0.14 & 0.14 & 0 & 0 & 0.76 & 0.63 & 0.32 & 0.14 & 0.45 & 0.47 & 0.12 & 0.12 \\
\hline
\end{tabular}

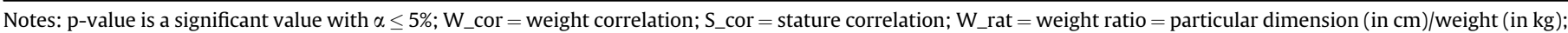

$\mathrm{S} \_$rat $=$stature ratio $=$particular dimension $($in $\mathrm{cm}) /$ stature $($ in $\mathrm{cm}) ; \mathrm{C}=$ Chinese; $\mathrm{NC}=$ non-Chinese; bolded figures show high correlation.

Table 9

Significant correlations and ratios with stature and weight for male and female subjects for elderly.

\begin{tabular}{|c|c|c|c|c|c|c|c|c|c|c|}
\hline \multirow[t]{3}{*}{ Dimension } & \multicolumn{10}{|c|}{ Elderly } \\
\hline & \multicolumn{2}{|c|}{ p-value } & \multicolumn{2}{|c|}{ W_Cor } & \multicolumn{2}{|c|}{ S_Cor } & \multicolumn{2}{|c|}{ W_Rat } & \multicolumn{2}{|l|}{ S_Rat } \\
\hline & $\mathrm{C}$ & NC & $\mathrm{C}$ & $\mathrm{NC}$ & $\mathrm{C}$ & $\mathrm{NC}$ & $\mathrm{C}$ & $\mathrm{NC}$ & $\mathrm{C}$ & $\mathrm{NC}$ \\
\hline \multicolumn{11}{|l|}{ Male } \\
\hline Eye height & 0 & 0 & 0.28 & 0.26 & 0.93 & 0.92 & 2.37 & 2.39 & 0.93 & 0.92 \\
\hline Shoulder height & 0 & 0 & 0.31 & 0.41 & 0.94 & 0.95 & 2.1 & 2.21 & 0.82 & 0.83 \\
\hline Elbow height & 0 & 0 & 0.35 & 0.34 & 0.87 & 0.83 & 1.85 & 1.98 & 0.61 & 0.64 \\
\hline Hip height & 0 & 0 & 0.36 & 0.43 & 0.81 & 0.79 & 1.54 & 1.64 & 0.56 & 0.55 \\
\hline Fingertip height & .01 & 0.03 & 0.21 & 0.44 & 0.79 & 0.91 & 1.1 & 1.12 & 0.37 & 0.37 \\
\hline Sitting eye height & 0 & 0 & 0.25 & 0.45 & 0.84 & 0.72 & 1.12 & 1.23 & 0.41 & 0.4 \\
\hline Span & 0 & 0 & 0.26 & 0.31 & 0.68 & 0.65 & 2.68 & 2.71 & 1.02 & 0.99 \\
\hline Vertical grip reach (standing) & 0 & 0 & 0.41 & 0.34 & 0.71 & 0.72 & 3.35 & 3.41 & 1.16 & 1.11 \\
\hline Thigh thickness & 0 & 0 & 0.73 & 0.69 & 0.41 & 0.34 & 0.26 & 0.28 & 0.07 & 0.08 \\
\hline Abdominal depth & 0 & 0 & 0.74 & 0.68 & 0.15 & 0.35 & 0.49 & 0.51 & 0.12 & 0.11 \\
\hline \multicolumn{11}{|l|}{ Female } \\
\hline Eye height & 0 & 0 & 0.56 & 0.29 & 0.95 & 0.93 & 2.31 & 2.35 & 0.94 & 0.97 \\
\hline Shoulder height & 0 & 0 & 0.54 & 0.51 & 0.91 & 0.88 & 1.99 & 1.98 & 0.83 & 0.84 \\
\hline Elbow height & 0 & 0 & 0.42 & 0.42 & 0.59 & 0.57 & 1.83 & 1.81 & 0.64 & 0.65 \\
\hline Hip height & 0 & 0 & 0.48 & 0.39 & 0.67 & 0.79 & 1.54 & 1.48 & 0.57 & 0.58 \\
\hline Fingertip height & .01 & 0 & 0.49 & 0.37 & 0.78 & 0.48 & 1.12 & 1.27 & 0.43 & 0.45 \\
\hline Sitting eye height & 0 & 0.03 & 0.32 & 0.41 & 0.65 & 0.68 & 1.1 & 1.13 & 0.45 & 0.51 \\
\hline Span & 0 & 0 & 0.39 & 0.39 & 0.81 & 0.83 & 2.71 & 2.76 & 1.03 & 1.04 \\
\hline Vertical grip reach (standing) & 0 & 0 & 0.27 & 0.31 & 0.74 & 0.75 & 3.41 & 3.45 & 1.18 & 1.19 \\
\hline Thigh thickness & 0 & 0 & 0.81 & 0.62 & 0.44 & 0.03 & 0.28 & 0.29 & 0.08 & 0.09 \\
\hline Abdominal depth & 0 & 0 & 0.76 & 0.64 & 0.31 & 0.12 & 0.48 & 0.51 & 0.15 & 0.16 \\
\hline
\end{tabular}

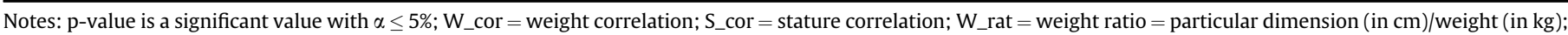

$\mathrm{S} \_$rat $=$stature ratio $=$particular dimension $($in $\mathrm{cm}) /$ stature $($ in $\mathrm{cm}) ; \mathrm{C}=$ Chinese; $\mathrm{NC}=$ non-Chinese; bolded figures show high correlation.

\section{Discussion and analysis}

This study has been done with a focus on special population, i.e., children and elderly. The children itself has been split into 2 parts, namely, (i) 6-9 years and (ii) 10-12 years. Related to elderly, it is critical as the numbers of aging people and their percentage in the overall population become larger, thus the need for complete, reliable, quantitative, and valid information about this population arises (Kroemer, 2006). Despite their physical limit, elderly people simply liking to do work, returning to work after raising children and enjoying longer lives. When it comes to the children anthropometry, especially at the range of 6-12 years, it deals with horizontal growth and gradual changes in physical appearance. Nowadays, facilities for children are ubiquitous, ranging from learning into playing devices.

A study on children and elderly anthropometry raises more attention due to its unique characteristics, thus, it is called as special population. The changes in body size especially during 
Table 10

Comparison of old and new anthropometric data.

\begin{tabular}{|c|c|c|c|c|c|c|c|c|c|c|c|c|c|c|c|c|}
\hline \multirow[t]{3}{*}{ Dimension } & \multicolumn{4}{|c|}{ Children $6-9$ years (New) } & \multicolumn{4}{|c|}{ Children $10-12$ years (New) } & \multicolumn{4}{|c|}{ Elderly (New) } & \multicolumn{2}{|c|}{ Adult (Hartono and Gunawan, 2015) } & \multirow{2}{*}{\multicolumn{2}{|c|}{$\frac{\text { Adult (Chuan et al., 2010) }}{\text { S_Rat }}$}} \\
\hline & \multicolumn{2}{|c|}{ W_Rat } & \multicolumn{2}{|c|}{ S_Rat } & \multicolumn{2}{|c|}{ W_Rat } & \multicolumn{2}{|l|}{ S_Rat } & \multicolumn{2}{|c|}{ W_Rat } & \multicolumn{2}{|c|}{ S_Rat } & \multirow{2}{*}{$\frac{\text { W_Rat }}{\text { Overall }}$} & \multirow[t]{2}{*}{ S_Rat } & & \\
\hline & $\mathrm{C}$ & NC & $\mathrm{C}$ & NC & $\mathrm{C}$ & NC & $\mathrm{C}$ & NC & $\mathrm{C}$ & NC & $\mathrm{C}$ & NC & & & $\mathrm{C}$ & Overall \\
\hline \multicolumn{17}{|l|}{ Male } \\
\hline Eye height & 4.55 & 3.77 & 0.86 & 0.91 & 3.43 & 3.12 & 0.91 & 0.9 & 2.37 & 2.39 & 0.93 & 0.92 & 2.36 & 0.93 & 0.93 & 0.93 \\
\hline Shoulder height & 4.05 & 3.37 & 0.77 & 0.82 & 3.04 & 2.76 & 0.81 & 0.8 & 2.1 & 2.21 & 0.82 & 0.83 & 2.09 & 0.83 & 0.83 & 0.83 \\
\hline Hip height & 2.66 & 2.30 & 0.5 & 0.56 & 2.08 & 1.87 & 0.55 & 0.54 & 1.54 & 1.64 & 0.56 & 0.55 & 1.42 & 0.56 & 0.55 & 0.55 \\
\hline Fingertip height & 1.77 & 1.50 & 0.33 & 0.36 & 1.37 & 1.19 & 0.36 & 0.35 & 1.1 & 1.12 & 0.37 & 0.37 & 0.94 & 0.37 & 0.37 & 0.37 \\
\hline Sitting eye height & 2.34 & 1.81 & 0.44 & 0.44 & 1.63 & 1.44 & 0.43 & 0.42 & 1.12 & 1.23 & 0.41 & 0.4 & 1.24 & 0.49 & 0.46 & 0.44 \\
\hline Thigh thickness & 0.46 & 0.34 & 0.09 & 0.08 & 0.29 & 0.29 & 0.08 & 0.08 & 0.26 & 0.28 & 0.07 & 0.08 & 0.22 & 0.08 & 0.1 & 0.1 \\
\hline $\begin{array}{l}\text { Abdominal depth } \\
\text { Female }\end{array}$ & 0.72 & 0.59 & 0.14 & 0.12 & 0.48 & 0.47 & 0.13 & 0.14 & 0.49 & 0.51 & 0.12 & 0.11 & 0.31 & 0.12 & 0.13 & 0.12 \\
\hline Eye height & 4.12 & 3.76 & 0.9 & 0.95 & 3.48 & 3.33 & 0.93 & 0.88 & 2.31 & 2.35 & 0.94 & 0.97 & 2.9 & 0.92 & 0.93 & 0.9 \\
\hline Shoulder height & 3.66 & 3.27 & 0.8 & 0.82 & 3.02 & 2.86 & 0.81 & 0.76 & 1.99 & 1.98 & 0.83 & 0.84 & 2.58 & 0.82 & 0.83 & 0.8 \\
\hline Hip height & 2.51 & 2.40 & 0.55 & 0.6 & 2.12 & 2.08 & 0.57 & 0.55 & 1.54 & 1.48 & 0.57 & 0.58 & 1.77 & 0.57 & 0.56 & 0.5 \\
\hline Fingertip height & 1.64 & 1.67 & 0.36 & 0.42 & 1.36 & 1.39 & 0.36 & 0.37 & 1.12 & 1.27 & 0.43 & 0.45 & 1.19 & 0.38 & 0.38 & 0.4 \\
\hline Sitting eye height & 2.02 & 1.91 & 0.44 & 0.48 & 1.61 & 1.71 & 0.43 & 0.45 & 1.1 & 1.13 & 0.45 & 0.51 & 1.43 & 0.46 & 0.46 & 0.5 \\
\hline Thigh thickness & 0.33 & 0.32 & 0.07 & 0.08 & 0.29 & 0.29 & 0.08 & 0.08 & 0.28 & 0.29 & 0.08 & 0.09 & 0.25 & 0.08 & 0.1 & 0.1 \\
\hline Abdominal depth & 0.62 & 0.56 & 0.14 & 0.14 & 0.45 & 0.47 & 0.12 & 0.12 & 0.48 & 0.51 & 0.15 & 0.16 & 0.37 & 0.12 & 0.13 & 0.1 \\
\hline
\end{tabular}

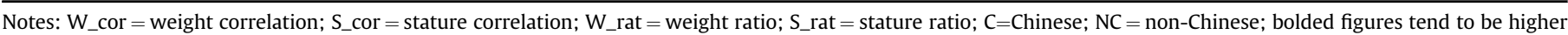
than other values at the same category of ethnic group.

childhood may vary from one individual to others, due to genetic factor, health, nutrition and environmental issues (Wall, 1993; Kroemer, 2006). Moreover, in general, body size increases rapidly during infancy (up to 2 years), and then more slowly until the start of puberty. In most males, growth accelerates at around 11 years, and reaches its peak at about 14 years, and then slows until adult stature is attained in the early to middle 20s. Whilst, for females, the fastest growth at around 12 years, and full adult stature is often complete at 16 years. Hence, a study on children anthropometry is of interest, especially how this dynamic growth fits the sociotechnical system.

After years, when it comes to aging, some may show rapid change in anthropometry, due to numerous alterations in musculoskeletal features such as bones become stiffer and more brittle, and shrinking of spinal discs that influence the height of limb segments.

Interestingly, comparing the Chinese and non-Chinese elderly sub-group, there was no significant difference, at both male and female group. It applied to the children anthropometry of 10-12 years sub-group at both male and female, as well. More interestingly, at the early stage of children growth (i.e., 6-9 years subgroup), it has been found that the non-Chinese group tend to have bigger dimensions than the Chinese one. In addition, the male and female children of 6-9 years (both Chinese and non-Chinese sub-group) had higher weight ratio, compared to elderly. It was easily recognized that children tend to have higher rate for limb segments compared to other body dimensions within their growing periods (Kroemer, 2006).

According to BMI, it is shown that both special populations (i.e., children and elderly, at both male and female samples) were located at the healthy weight zone (18.5-24.99), except for male and female Chinese children of 6-9 years sub-group (i.e., 16.1 and 17.9, respectively). Though, this value is not far away from the healthy range. According to WHO (2017), those BMI are still acceptable as they are within the healthy BMI range (i.e., around 14-19).

More interestingly, both female and male elderly for Chinese and non-Chinese samples showed BMI nearly the upper limit of 24.99. They may have gained more weight due to less exercise and activity. Moreover, within the age bracket, some elderly show rapid change in anthropometry within few years, for instance, in stature because of posture and shrinking of spinal discs, and in weight because of changes in nutrition, metabolism, and health (Kroemer, 2006).

In general, the male children tend to have bigger body dimensions than the female one. Apart from what have been known that male children grow faster at about 11 (Kroemer, 2006), it may be influenced by better physical activities. Compared to others, male children do more physical activities and exercises.

In general, stature was highly correlated with eye height, shoulder height, elbow height, hip height, fingertip height, sitting eye height, span, and vertical grip reach (standing) in all population groups. More specifically, there were two measures (i.e., eye height and shoulder height) can be confidently predicted by a given stature. Related to body weight, thigh thickness and abdominal depth were deemed to be closely correlated with. The finding shows that, with a very limited time or other resources (e.g., the number of subjects), several body dimensions of a particular population were able to be predicted confidently, given a precise stature or body weight. Inherently, stature and body weight are basic measures, and easy to collect by layman or less experienced person. Even, a simple meter tape can be used for measuring stature, and a simple weighing scale can be utilized (Hartono and Gunawan, 2015).

The data set of anthropometric body dimensions are utilized to design specific products considering these two different special populations. At the elderly group, the targeted products may include office chairs and tables, bedroom and dining room furniture, kitchen tops and storage space, placement of electric switches, door handles, and windows (Kothiyal and Tettey, 2001). At the children group in the school age, according to Saptari et al. (2013), the products designed may cover backpacks, toys, desks, chairs, and writing utensils.

\section{Conclusion, limitation and further research}

Given more valid procedures and sufficient sample size, the human body dimensions can be predicted in the near future by proposing mathematical modeling, which is revisiting the Drillis and Contini's ratio (1966), and has been proposed by Peacock et al. (2012).

Following the previous research (see Peacock et al., 2012; Hartono, 2015), this study confirms the findings that body weight was found to be a predictor of width and girth measures, whereas stature was found to be highly correlated with limb lengths. More 
specifically, this study provides more insight about which body segments can be predicted more precisely through either stature or body weight, according to statistical testing. Practically, the findings can be applied to any product or system design taking into account anthropometric measures, especially for special populations (i.e., children and elderly).

However, this study is limited and far from complete. The generalization of the findings from the study is limited due to the relatively small sample size. In this study, the relative small sample size is shown at the Chinese male children anthropometric data. Furthermore, the study on other special population settings should be of interest, such as pregnant woman, diffability, and ethnic group-based Indonesian anthropometry.

For further studies, it is essential to include and take into account unique ethnic groups for Indonesian. Major ethnic groups within different special populations may be of interest, such as Javanese, Madurese and Balinese. Another factor such as geographical such as Papua will be of interest. Papua people have different face and posture, compared to Indonesian majority. It may due to different region where they live, i.e., more mountainous and forest areas. Another interesting potential is that how to validate the linear model and apply the sufficient anthropometric data into products. Moreover, more information about individual differences on anthropometry (Kroemer, 2006) is of interest. Anthropometry demands strict timelineness. According to Hu et al. (2015), largescale anthropometric measurement study should be conducted every 10 years.

\section{Acknowledgment}

This research was fully financially supported by the Department of Industrial Engineering-University of Surabaya, and the Indonesian Directorate General of Higher Education through the Competitive Grant ("Hibah Bersaing") year 2015 under a contract number “015/SP-Lit/LPPM-01/Dikti/FT/IV/2015”.

\section{Appendix A. Supplementary data}

Supplementary data related to this article can be found at https://doi.org/10.1016/j.ergon.2018.01.004.

\section{References}

Al-Ansari, M., Mokdad, M., 2009. Anthropometrics for the design of Bahraini school furniture. Int. J. Ind. Ergon. 39, 728-735.

Altares, P.S., Copo, A.R.I., Gabuyo, Y.A., Laddaran, A.T., Mejia, L.D.P., Policarpio, I.A., Sy, E.A.G., Tizon, H.D., Yao, A.M.S.D., 2003. Elementary Statistics: a Modern Approach, first ed. Rex Book Store, Inc., Manila.

Babbie, E., 1992. The Practice of Social Research, sixth ed. Wadsworth, Belmont, CA.

Bombais, D.V., Ferrer, J.A., Perea, A.P., Portus, A.J., 2017. Ergonomic assessment and evaluation of Philippine buses for Filipinos: a case study on metro manila city buses. In: Stanton, N., Landry, S., Di Bucchianico, G., Vallicelli, A. (Eds.), Advances in Human Aspects of Transportation. Advances in Intelligent Systems and Computing, vol. 484. Springer, Cham.

BPS, 2015. Indonesian Central Bureau of Statistics available online at: https://www. bps.go.id.

Barroso, M.P., Arezes, P.M., da Costa, L.G., Miguel, A.S., 2005. Anthropometric study of Portuguese workers. Int. J. Ind. Ergon. 35, 401-410.

Bolstad, G., Benum, B., Rokne, A., 2001. Anthropometry of Norwegian light industry and office workers. Appl. Ergon. 32, 239-246.

Cadman, D., Boyle, M., Szatmari, P., Offord, D.R., 1987. Chronic illness, disability, and mental and social well-being: findings of the Ontario child health study. Pediatrics 79, 805-813.

Chuan, T.K., Hartono, M., Kumar, N., 2010. Anthropometry of the Singaporean and Indonesian populations. Int. J. Ind. Ergon. 40, 757-766.

Del Prado-Lu, J.L., 2007. Anthropometric measurement of Filipino manufacturing workers. Int. J. Ind. Ergon. 37, 497-503.

Drillis, R., Contini, R., 1966. Body Segment Parameters, DHEW 1166-03. New York University, School of Engineering and Science.

Gavin, M.L., 2015. Growth and Your 6- to 12-year-old [Online] Available from: http://kidshealth.org/en/parents/growth-6-12.html\#. (Accessed 14 August
2017).

Ghaderi, E., Maleki, A., Dianat, I., 2014. Design of combine harvester seat based on anthropometric data of Iranian operators. Int. J. Ind. Ergon. 44, 810-816.

Gravetter, F.J., Forzano, L.-A.B., 2012. Research Methods for the Behavioral Sciences Linda Schreiber-Ganster, Wadsworth, Cengage Learning, Belmont, CA, USA.

Hanson, L., Sperling, L., Gard, G., Ipsen, S., Vergara, C.O., 2009. Swedish anthropometrics for product and workplace design. Appl. Ergon. 40, 797-806.

Harih, G., Dolšak, B., 2013. Tool-handle design based on a digital human hand model. Int. J. Ind. Ergon. 43 (4), 288-295.

Hartono, M., 2015. Drillis \& Contini revisited using structural equation modeling for anthropometric data. In: Seminar Dan Kongres Nasional Perhimpunan Ergonomi Indonesia, 17-19 November 2015, Yogyakarta, Indonesia.

Hartono, M., Gunawan, L., 2015. Drillis and Contini revisited using correlation analysis for Indonesian adults anthropometry. In: IEEE Industrial Engineering \& Engineering Management (IEEM) Conference, 6-9 September 2015, Singapore.

Hartono, 2016. Indonesian anthropometry update through Drillis \& Contini revisited and structural equation modeling incorporating children, adult and elderly populations. In: Proceeding of IEEE Industrial Engineering and Engineering Management (IEEE IEEM) International Conference 4-7 December 2016 in Bali, Indonesia, pp. 262-266.

Helander, M., 1997. Forty years of IEA: some reflections on the evolution of ergonomics. Ergonomics 40, 952-961.

Hu, H.-M., Zhao, C.-Y., Zhang, X., Ran, L.-H., Liu, T.-J., 2015. Correlation analysis on the main and basic body dimension for Chinese adults, in digital human modeling: applications in health, safety, ergonomics and risk management: ergonomics and health. In: Duffy, V.G. (Ed.), 6th International Conference, DHM 2015, Held as Part of HCI International 2015, Los Angeles, CA, USA, August 2-7, 2015.

Jarosz, E., 1999. Anthropometry of elderly women in Poland: dimensions for design Int. J. Ind. Ergon. 25, 203-213.

Jones, A.D., Ickes, S.B., Smith, L.E., Mbuya, M.N.N., Chasekwa, B., Heidkamp, R.A. Menon, P., Zongrone, A.A., Stoltzfus, R.J., 2014. World Health Organization infant and young child feeding indicators and their associations with child anthropometry: a synthesis of recent finding. Matern. Child Nutr. 10, 1-17. https:// doi.org/10.1111/mcn.12070.

Khadem, M.M., Islam, M.A., 2014. Development of anthropometric data for Bangladeshi male population. Int. J. Ind. Ergon. 44, 407-412.

Klamklay, J., Sungkhapong, A., Yodpijit, N., Patterson, P.E., 2008. Anthropometry of the southern Thai population. Int. J. Ind. Ergon. 38, 111-118.

Kothiyal, K., Tettey, S., 2001. Anthropometry for design for the elderly. Int. J. Occup. Saf. Ergon. 7 (1), 15-34.

Kroemer, K.H.E., Grandjean, E., 1997. Fitting the Task to the Human: a Textbook of Occupational Ergonomics, fifth ed. CRC Press.

Kroemer, K.H.E., 2006. Extra-ordinary Ergonomics: How to Accommodate Small and Big Persons, the Disabled and Elderly, Expectant Mothers, and Children. CRC Press, Taylor \& Francis Group, Boca Raton, FL.

Leslie, F., Marko, K., Christopher, F., 2005. International anthropometric study of facial morphology in various ethnic groups/races. J. Craniofac. Surg. 16, 615-646.

McCrory, J., Harrison, K., Mancinelli, C., Meszaros, P., Thomas, K., 2016. The effect of added weight on foot anthropometry in pregnant women and controls. Int. J. Exerc. Sci. Conf. Proc. 9 (4), 70.

Mokdad, M., 2002. Anthropometric study of Algerian farmers. Int. J. Ind. Ergon. 29 $331-341$.

Montgomery, D.C., Runger, G.C., Hubele, N.F., 2010. Engineering Statistics: Student Study Edition, fourth ed. John Wiley \& Sons Inc.

Olafsdottir, S., Berg, C., Eiben, G., Lanfer, A., Reisch, L., Ahrens, W., Kourides, Y., Molnár, D., Moreno, L.A., Siani, A., Veidebaum, T., Lissner, L., 2014. Young children's screen activities, sweet drink consumption and anthropometry: results from a prospective European study. Eur. J. Clin. Nutr. 68, 223-228. https:// doi.org/10.1038/ejcn.2013.234.

Peacock, J.B., Aravindakshan, M., Xin, T., Ping, C.Y., Ping, L.W., Ding, F., Chuan, T.K. Hartono, M., Stella, N.Y., 2012. Drillis and Contini revisited. In: Rebelo, F. Soares, M.M. (Eds.), Advances in Usability Evaluation. CRC Press, Boca Raton.

Pennathura, A., Dowling, W., 2003. Effect of age on functional anthropometry of older Mexican American adults: a cross-sectional study. Int. J. Ind. Ergon. 32, 39-49.

Pheasant, S., Haslegrave, C.M., 2006. Body Space: Anthropometry, Ergonomics and the Design of Work, third ed. Taylor \& Francis, Inc., New York.

Pitt, L.F., Watson, R.T., Kavan, C.B., 1995. Service quality: a measure of information systems effectiveness. MIS Q. 19 (2), 173-187.

Sadeghi, F., Mazloumi, A., Kazemi, Z., 2015. An anthropometric data bank for the Iranian working population with ethnic diversity. Appl. Ergon. 48, 95-103.

Saptari, A., Ng, P.K., Mukhyi, M.M., 2013. The importance of child anthropometry in child product designs. In: Deros, Baba Md, Yusuff, Rosnah Mohd, Daruis, Dian Darina Indah, Mohamad, Darliana, Yusoff, Ahmed Rithauddeen (Eds.), Anthropometric Research in Malaysia, Chapter: 5. National Institute of Occupational Safety and Health (NIOSH), Malaysia.

Temple, V.A., Foley, J.T., Lloyd, M., 2014. Body mass index of adults with intellectual disability participating in Special Olympics by world region. J. Intellect. Disabil. Res. 58, 277-284. https://doi.org/10.1111/jir.12011.

Tinetti, M.E., 1986. Performance-oriented assessment of mobility problems in elderly patients. J. Am. Geriatr. Soc. 34, 119-126.

Victor, V.M., Nath, S., Verma, A., 2002. Anthropometric survey of Indian farm workers to approach ergonomics in agricultural machinery design. Appl. Ergon. 
33, 579-581.

Wall, H.A.D., 1993. Environmental factors influencing growth and pubertal development. Environ. Health Perspect. Suppl. 101, 39-44.

Wickens, C.D., Lee, J.D., Liu, Y., Gordon-Becker, S.E., 2004. An Introduction to Human Factors Engineering, second ed. Pearson Education, Upper Saddle River, New Jersey.

Widyanti, A., Susanti, L., Sutalaksana, I.Z., Muslim, K., 2015. Ethnic differences in Indonesian anthropometry data: evidence from three different largest ethnics. Int. J. Ind. Ergon. 47, 72-78.

Widyanti, A., Mahachandra, M., Soetisna, H.R., Sutalaksana, I.Z., 2017.
Anthropometry of Indonesian Sundanese children and the development of clothing size system for Indonesian Sundanese children aged 6-10 year. Int. J. Ind. Ergon. 61, 37-46.

World Health Organization (WHO), 2016. Body Mass Index (BMI) Classification [Online] Available from: http://apps.who.int/bmi/index.jsp?introPage=intro_3. html. (Accessed 17 October 2016).

World Health Organization (WHO), 2017. Body Mass Index (BMI) for Age 5-19 Years [Online] Available from: http://www.who.int/growthref/who2007_bmi_for_a ge/en. (Accessed 14 April 2017). 